\title{
R-loop-induced p21 expression following CDC73, CTR9, and PAF1 loss protects cancer cells against replicative catastrophe following WEE1 inhibition
}

Linda van Bijsterveldt ${ }^{1}$, Helga B. Landsverk ${ }^{2}$, Viola Nähse ${ }^{2}$, Samuel C. Durley ${ }^{1}$, Sovan S. Sarkar ${ }^{1}$, Randi G. Syljuåsen ${ }^{2}$, and Timothy C. Humphrey ${ }^{1}$

${ }^{1}$ CRUK MRC Oxford Institute for Radiation Oncology, Department of Oncology, University of Oxford, ORCRB, Roosevelt Drive, Oxford OX3 7DQ, UK

${ }^{2}$ Department of Radiation Biology, Institute for Cancer Research, Norwegian Radium Hospital, Oslo University Hospital, Oslo, Norway

\begin{abstract}
WEE1 inhibitors have now advanced into clinical studies as monotherapy or in combination with chemoradiotherapy in TP53, RAS, BRAF, and SETD2 mutation carriers across several tumour types, yet mechanisms of resistance are still poorly understood. Here, we further elucidate the mechanisms by which AZD1775, the most potent WEE1 inhibitor, kills cells and reveal additional genetic interactions that can result in resistance, but could be used to optimise its clinical utility.
\end{abstract}

We identified RNA Polymerase II-associated factor 1 (PAF1) complex members, CDC73, CTR9, and PAF1 as major determinants of WEE1-inhibitor sensitivity in isogenic SETD2-positive and negative cell lines. PAF1-knockdown cells resist higher doses of the WEE1 inhibitor, which we show is due to reduced DNA damage induction $(\gamma \mathrm{H} 2 \mathrm{AX})$ and delayed $\mathrm{G} 1$ checkpoint activation, ultimately protecting cells against replicative catastrophe. Investigations into the molecular mechanisms responsible for PAF1-mediated resistance identify involvement of Rloops and subsequent activation of the cyclin-dependent kinase inhibitor p21Cip1/Waf1, which in addition to causing prolonged $\mathrm{G} 1$ arrest in the following cell cycle, also regulates CDK activity, therefore limiting replication.

These results provide evidence that the PAF1 complex and p21 are important regulators of proliferation under increased DNA replication stress and their expression levels might be useful biomarkers to predict clinical response to WEE1 inhibitors and other ribonucleotide reductase inhibitors.

\section{Introduction}

The use of cell cycle checkpoint kinase inhibitors, including WEE1 inhibitors, has emerged as a promising targeted strategy aimed to exploit cancer cells with a dysfunctional G1/S checkpoint (van Bijsterveldt et al., 2020). Several WEE1 inhibitors have been developed pre-clinically (Matheson, Backos and Reigan, 2016), and a number of those have now advanced into 
clinical studies as monotherapy or in combination with chemoradiotherapy in TP53, RAS, and $B R A F$ mutation carriers across several tumour types (Rorà et al., 2020). WEE1 kinase is responsible for inhibitory tyrosine-15 (Y15) phosphorylation of the ATP-binding domain of cyclin dependent kinase 1 and 2 (CDK1/2) (Parker and Piwnica-Worms, 1992). Accordingly, inhibition of WEE1 results in CDK1 and CDK2 hyperactivation during S-phase, which promotes increased replication origin firing as well as degradation of RRM2, the regulatory subunit of ribonucleotide reductase (RNR) that catalyses the rate-limiting step of the formation of deoxyribonucleotides (dNTPs) (D'Angiolella et al., 2012; Pfister et al., 2015).

Our group has recently demonstrated that SETD2-deficient cancers, with SETD2 encoding the only histone methyltransferase that catalyses H3 lysine 36 trimethylation (H3K36me3) (Edmunds, Mahadevan and Clayton, 2008), can be targeted by exploiting a synthetic lethal interaction with WEE1 inhibition (WEE1i) by AZD1775 (Pfister et al., 2015). SETD2-deficient cells display lower RRM2 transcript levels and can therefore be preferentially targeted with the potent WEE1 inhibitor AZD1775 as dNTP pools reach critically low levels (Pfister et al., 2015). Reduced intracellular dNTP pools in this context provoke catastrophic widespread fork collapse and accumulation of DNA double-strand breaks (DSBs), ultimately leading to replicative catastrophe (Poli et al., 2012; Pfister et al., 2015). Therefore, cell death resulting from WEE1 inhibition in combination with the loss of SETD2 is thought to be primarily caused by replicative catastrophe (Pfister et al., 2015; Pai et al., 2019). Even though synthetic lethalitybased cancer therapies have been proven to be highly effective, therapy outcomes may not be durable due to recurrent patterns of resistance development within tumours. In the case of WEE1 inhibition, it remains unclear whether processes beyond canonical checkpoint control can modulate response and resistance to WEE1 inhibitor treatment, which is why there is a pressing need to identify clinically relevant biomarkers of sensitivity.

The RNAPII-associated factor 1 (hPAF1) complex, is a positive elongation factor and is also required for chromatin modifications, particularly ubiquitination of $\mathrm{H} 2 \mathrm{~B} \quad \mathrm{~K} 120$ and $\mathrm{H} 3 \mathrm{~K} 4$ methylation (Nevan J. Krogan et al., 2003; $\mathrm{Ng}$ et al., 2003; Wood et al., 2003). In S. cerevisiae and $S$. pombe but not mammalian cells, recruitment of Set2 and H3K36 methylation to actively transcribed genes relies on components of the yeast Paf1 complex, as well as the RNAPII CTD kinase Ctk1 (ortholog of CDK12) in $S$. cerevisiae and cyclin-dependent kinase 9 (Cdk9) in S. pombe (Nevan J Krogan et al., 2003; Mbogning et al., 2013). Our group has identified PAF1 and PRF1 (assigned RTF1 in mammalian cells) (Vos et al., 2020), two components of PAF1C, as two genes putatively responsible for suppression of the set2A wee1-50 synthetic lethality using a suppressor screen. The human PAF1C mainly functions to release paused RNAPII from the promoter-proximal pause site (20-60 nucleotides downstream of the transcription start site (TSS)) into effective transcriptional elongation as it displaces negative elongation factor (NELF) from the RNAPII funnel (Chen et al., 2015; Yu et al., 2015; Vos et al., 2018). Inactivation of the PAF1 complex leads to RNAPII accumulation at the 
proximal-promoter pausing site in both budding yeast (Wahba et al., 2011) and mammalian cells, causing unscheduled R-loop formation (Shivji et al., 2018) and checkpoint activation. To prevent the genome against R-loop associated DNA damage, unscheduled R-loop accumulation can activate the ATR-Chk1 signalling pathway in a replication-dependent manner (Matos et al., 2020), thereby enforcing checkpoint arrest, suppression of transcription-replication conflicts, and promoting fork recovery. Dysregulation of R-loops can cause genomic instability, one of the enabling characteristics of cancer, via formation of DSBs, chromosome breaks, and recombination events (Hanahan and Weinberg, 2011; Crossley, Bocek and Cimprich, 2019).

Here, we find that loss of paf1 in fission yeast suppresses the set2 $\Delta$ wee1-50 synthetic lethality. Furthermore, depletion of PAF1C members, including CDC73, CTR9 and PAF1 in human osteosarcoma (U2OS) cells results in resistance to cell death following WEE1 inhibition in both the presence and absence of SETD2. Depletion of PAF1C members results in an increase in p21, which binds to and inhibits both cyclin-CDK complexes and proliferating nuclear antigen (PCNA). The upregulation of p21, via its ability to induce the G1 checkpoint following DNA damage, prevents replication and protects cells from cell death following WEE1 inhibition. These results are of importance to guide the future clinical implementation of the WEE1 inhibitor, as tumours with low p21 expression as a result of transcriptional dysregulation or TP53 deficiency may be particularly sensitive, whereas tumours with mutations in CTR9, $P A F 1, C D C 73$, or other cancers with mutations up-regulating p21 levels might prove to be resistant to ribonucleotide reductase inhibitor treatment.

\section{Results}

Loss of transcription elongation factor Paf1 rescues the synthetic lethality resulting from Set2 deletion and Wee1 inactivation in S. pombe

To explore the possible mechanisms of resistance to WEE1 inhibitor treatment in H3K36me3deficient cells, a genetic screen was performed in fission yeast to isolate spontaneous mutants that suppressed the synthetic lethal interaction between set2 $\Delta$ and wee 1-50, a temperaturesensitive wee1, which is inactive when grown at the restrictive temperature of $35.5^{\circ} \mathrm{C}$ (Russell and Nurse, 1987). Of approximately 10,000 set24 wee1-50 cells plated, 23 mutants were identified which were able to grow at $35.5^{\circ} \mathrm{C}$. Sequence analysis of these mutants included loss of function mutations in $c d c 2$, as expected, and a number of additional mutants, including mutations in seven genes involved in transcription, one of which was paf1, a subunit of the polymerase associated factor complex.

To validate the results of the screen, we generated set2 $\Delta$ wee1-50 paf1 $\Delta$ and tested their ability to form colonies using a serial dilution assay (Figure $1 \mathrm{~A}$ ). Growth of set2 $\Delta$ wee1-50 was compromised when grown at semi-permissive $\left(32^{\circ} \mathrm{C}\right)$ temperature (Figure $1 \mathrm{~A}$ ) as previously described (Pai et al., 2019). Deletion of paf1 ${ }^{+}$, however, suppressed the set2A wee1-50 
synthetic lethality, as the set2 $\Delta$ wee1-50 paf1 triple mutants were able to grow at $32{ }^{\circ} \mathrm{C}$ in contrast to set2 $\Delta$ wee1-50 double mutants (Figure $1 \mathrm{~A}$ ).

\section{Knockdown of PAF1, CTR9, or CDC73 desensitises U20S cells to WEE1-inhibition}

To investigate whether Paf1-mediated suppression of Wee1 inactivation is conserved from fission yeast to human cells, PAF1 was silenced by RNA interference in SETD2 WT and SETD2 CRISPR knockout (KO) human osteosarcoma (U20S) cell lines and sensitivity to the WEE1-inhibitor AZD1775 was measured. Knockdown of PAF1 was confirmed by Western Blot (Supplemental Figure 1A). Knock-down of PAF1 significantly restored cell viability in the presence 150, 300 and $600 \mathrm{nM}$ of AZD1775 (Figure 1B-C). The survival rate at maximum inhibition (Imax\% value) differed significantly between non-targeting control siRNA and PAF1 siRNA treated U2OS cells, with a stronger effect observed in SETD2 CRISPR KO U2OS cells (non-targeting siRNA control $I_{\max \%}=6.36$ versus PAF1 siRNA $I_{\max \%}=44.7$, $p$-value= 0.0082) (Figure 1B-C).

To exclude the possibility that U20S cells have acquired other unaccounted mutations that may contribute to the resistant phenotype observed following PAF1 knock-down, we also silenced PAF1 in the renal cell carcinoma (RCC) cell lines 786-0 and A498 (bearing a truncation mutation in SETD2), where WEE1-inhibitor sensitivity was measured (Supplemental Figure 1B). Consistent with our previous results, knock-down of PAF1 significantly desensitised 786-O and SETD2-mutant cell line A498 to AZD1775 (Supplemental Figure 1B). Increased cell viability following AZD1775 treatment in siPAF1-treated SETD2 CRISPR KO U2OS was, however, not accompanied by caspase-3-mediated-apoptosis resistance (Supplemental Figure 1C). The increased cell viability following PAF1 $K D$ is therefore not likely to result from resistance to cell death induction by apoptosis.

To further characterise the role of the PAF1 complex in mediating resistance to WEE1 inhibition, siRNA mediated gene-silencing was used to assess the effect of depletion of individual PAF1 complex members on AZD1775-sensitivity in both SETD2 WT and SETD2 KO U2OS cells. Knock-down of CDC73 and CTR9 phenocopied the effect of PAF1 knockdown (Figure 1D-E) following WEE1-inhibitor treatment, whereas silencing of LEO1 and RTF1 did not have a significant effect on cell viability (data not shown). Knockdown of PAF1 complex components was validated using Western Blot (Supplemental Figure 1D). We can therefore conclude that WEE1-inhibitor sensitivity is dependent on the presence of at least a subset of PAF1 complex members, including CDC73, CTR9, and PAF1.

Moreover, to investigate the importance of the PAF1C in regulating the cellular response to replication stress, we analysed the sensitivity patterns to hydroxyurea (HU) and gemcitabine following PAF1 depletion in SETD2 WT and SETD2 CRISPR KO U2OS cells. Our results indicate that PAF1 depletion renders U2OS cells less sensitive to increasing concentrations of 
hydroxyurea $(\mathrm{HU})$ and gemcitabine (Figure $1 \mathrm{~F}$ ), all of which directly deplete dNTP pools and subsequently block replication fork progression (Alvino et al., 2007; Cerqueira, Fernandes, \& Ramos, 2007). Similarly, in S. pombe, a spot assay showed that paf1 and $c d c 73 \Delta$ display reduced sensitivity to high concentrations of $\mathrm{HU}$ when compared to a wild-type strain (Figure 1G). We therefore conclude that loss of PAF1C components confers an evolutionarily conserved cross-resistance among inhibitors of ribonucleotide reductase.

\section{Loss of PAF1 rescues AZD1775-induced replicative catastrophe through damage reduction}

As previously described, AZD1775 leads to selective killing of SETD2 KO U20S cells through dNTP pool depletion, accumulation of non-replicating S-phase cells (exhibiting a DNA content between $2 \mathrm{~N}$ and $4 \mathrm{~N}$, but not incorporating the synthetic nucleoside bromodeoxyuridine [BrdU]) and, ultimately leading to replicative catastrophe (Pfister et al., 2015). Therefore, we tested whether loss of PAF1 restored cell viability by alleviating AZD1775-induced S-phase effects, such as reduced BrdU incorporation, indicative of slow S-phase progression, and DNA damage.

To examine the progression through S-phase following PAF1 silencing in SETD2 WT and SETD2 CRISPR KO U2OS, cells were treated with $300 \mathrm{nM}$ AZD1775 for 48 hours, pulselabelled with BrdU for $30 \mathrm{~min}$ and collected for cell cycle analysis. AZD1775 treatment resulted in accumulation of $50 \pm 0.3 \%$ of the SETD2 CRISPR KO U2OS cells in non-cycling Sphase, with only $16 \%$ in G1 (Figure 2A-B). In siPAF1-treated SETD2 CRISPR KO U2OS cells, despite inhibition of WEE1, only $26.4 \pm 3.8 \%$ of cells were in non-replicating S-phase, whereas the fraction of cells in G1 phase of the cell cycle was increased (38.6 $\pm 2.2 \%$ ) (Figure 2A-B).

To uncover the molecular mechanisms by which PAF1 depletion reduces the toxic effects of WEE1i in SETD2 KO U20S cells, we assessed DNA damage, using an antibody to $\gamma \mathrm{H} 2 \mathrm{AX}$, following WEE1 inhibitor treatment in S-phase U20S cells. Firstly, we found that replicative catastrophe resulting from AZD1775-treatment is a direct function of DNA damage. AZD1775 treatment $(1 \mu \mathrm{M})$ resulted in the accumulation of two distinct populations, a high $(16.1 \%)$ and an intermediate $(21.7 \%) \gamma \mathrm{H} 2 \mathrm{AX}$ population as early as 6 hours post-treatment (Supplemental Figure 2A). FACS analysis of DNA damage 4 hours following WEE1 inhibitor wash-out showed a clear reduction in the intermediate $\gamma \mathrm{H} 2 \mathrm{AX}$ population (reduction of $15.5 \%$ ), but no change in the percentage of cells with the highest levels of damage (Supplemental Figure 2B), suggesting that some of the DNA damage breaks might be irreversible, or take longer to repair. When the high $\gamma H 2 A X$ population was gated (indicated in black in the EdU plot, Supplemental Figure 2A), we found that the high $\gamma \mathrm{H} 2 \mathrm{AX}$ population was EdU-negative, suggestive of replication arrest (Supplemental Figure 2B).

To test whether the alleviation of replicative catastrophe following PAF1 depletion was a direct result of reduced DNA damage $(\gamma \mathrm{H} 2 \mathrm{AX})$ in S-phase, we compared $\gamma \mathrm{H} 2 \mathrm{AX}$ levels 48 hours following AZD1775-treatment $(250 \mathrm{nM})$ in siPAF1 treated U20S with control siRNA treated 
cells. We found that depleting PAF1 resulted in significantly reduced levels $(p=0.0038)$ of $\gamma \mathrm{H} 2 \mathrm{AX}$-high cells $(6.85 \%)$ compared to siNT-treated SETD2 KO U20S cells (10.36\%) following addition of the WEE1-inhibitor AZD1775 (Figure 2C-D). Furthermore, as shown in Figure 2E (quantified in Supplemental Figure $2 \mathrm{C}$ ), we found that levels of phosphorylated ATM, DNA-PK and RPA32 were also reduced following AZD1775 treatment in SETD2 KO U20S cells treated with siPAF1 when compared to the control-treated cells. This suggests reduced or delayed formation of replication-associated DNA DSBs (Liaw, Lee and Myung, 2011) and altered damage signalling in the absence of PAF1.

Finally, we assessed the effect of depleting other PAF1C components on the non-replicating S-phase population following WEE1 inhibition and found that depletion and CDC73 and CTR9 resulted in a similar reduction in the non-replicating S-phase population. When U20S cells depleted for CDC73 or CTR9 were treated with AZD1775 for 48 hours, we also observed an accumulation of cells in G1 and a decrease in S-phase population, with an effect on the rate of DNA synthesis with lower BrdU incorporation $(22.6 \%$ and $21.7 \%$ BrdU-negative Sphase cells for CDC73 and CTR9, respectively), when compared to the control- siRNA treated SETD2 CRISPR KO U20S cells (47.7\%) (Figure 2F).

Taken together, these results show that depletion of CDC73, CTR9 or PAF1 result in a larger fraction of cells in G1 phase following AZD1775 treatment, as well as a reduction in the non-replicating S-phase fraction, due to a lower percentage of cells with median and high $\gamma \mathrm{H} 2 \mathrm{AX}$ levels, leading to an increase in cell viability in the context of WEE1 inhibition.

\section{R-loop and P53-dependent up-regulation of CDKN1A following loss of CDC73, CTR9, and PAF1}

To further investigate the proliferation defect observed after CDC73, CTR9, or PAF1 depletion, siRNA-transfected U2OS cells were synchronised at the G1/S border by double thymidine block followed by release and assessment of cell cycle progression into S-phase by flow cytometry (Supplemental Figure $3 \mathrm{~A}-\mathrm{B}$ ). Consistent with the growth assays (Supplemental Figure 2), FACS analysis indicated that SETD2 WT U2OS cells depleted of PAF1, CDC73 or CTR9 spent more time in $\mathrm{G} 1$ after release than siNT-treated control cells (Figure 3A-B, Supplemental Figure $3 \mathrm{~A}-\mathrm{B}$ ). These data prompted us to examine the relationship between $\mathrm{p} 21$, a wellstudied regulator of the G1/S transition, and components of the PAF1C.

We found that the reduction in long-term colony formation of cells depleted for PAF1 (data not shown) coincided with increased mRNA and protein levels of p21 (Figure 3A-B), a potent inhibitor of Cdk-Cyclin complexes. We do, however, not observe any p21 expression in the 
absence of PAF1 in TP53 KO U2OS cells (data not shown). Following loss of PAF1, we found that elevated levels of p21 did indeed reduce CDK activity, as shown by a decrease in phosphorylation of CDK motifs for selected putative substrates, both in the absence and presence of AZD1775 (48 hours post-drug treatment) (Figure 3C). In agreement with this, we find that knockdown of PAF1 restores protein levels of RRM2 following WEE1-inhibitor treatment, consistent with reduced CDK-dependent RRM2 degradation (Supplementary Figure 3C). The above observations suggest that SETD2-CRISPR cells depleted for CDC73, CTR9 or PAF1 exhibit increased p21 levels, reduced CDK1/2 activity and delayed G1-S progression.

Loss of PAF1 has previously been shown to cause an increase in the formation of RNADNA hybrids (R-loops) (Shivji et al., 2018), which could lead to subsequent checkpoint signalling and p53 activation. Therefore, we asked whether up-regulation of p21, which leads to p53-dependent resistance to WEE1i, could be caused by R-loop formation in the absence of PAF1. First, global R-loop levels were assessed following depletion of SETD2 and/or PAF1 by a slot blot using the anti-DNA:RNA hybrid S9.6 antibody (Figure 3D). This showed an increase in overall R-loop levels in SETD2-, PAF1- and SETD2- and PAF1-depleted U2OS cells. As a control, we pre-treated the genomic DNA with RNase $\mathrm{H}$, which completely abolished the R-loop signal in both control and PAF1-/SETD2- knockdown samples cells (Figure 3D). To test whether the R-loops formed in cells depleted of PAF1 do indeed contribute to DNA damage and p53-mediated activation of p21, we transiently over-expressed V5-RNaseH1, which resolves R-loops, in control- and siPAF1 treated cells (Figure 3E). By analysing p21 levels, we found that expression of RNase $\mathrm{H} 1$, as shown by high levels of the V5-epitope tag, resulted in reduction of p21 levels (Figure $3 \mathrm{E}$ ). Our data suggest that elevated R-loops in the absence of SETD2 or PAF1 cause the observed changes in p53-pathway activation, ultimately leading to up-regulation of p21 and G1 arrest.

\section{Direct role for p21 in regulating sensitivity to WEE1 inhibitor treatment}

Next, we sought to determine whether this p21-mediated G1-S delay may be responsible for the observed resistance to WEE1 inhibition in the absence of PAF1. We reasoned that if the G1 delay was mediated by the p53-p21 pathway, then co-depletion of p21 should suppress the cell cycle delay induced by CDC73, CTR9 or PAF1 depletion. We found that depletion of p21 (CDKN1A) alone further increased the $\mathrm{BrdU}^{+}$and $\mathrm{BrdU}^{-}$S-phase population $(15.2 \%$ increase in S-phase compared to siNT-treated SETD2 KO U20S cells) following AZD1775 treatment, in accordance with the published role for p21 in regulating the G1/S transition (Abbas and Dutta, 2009) (Supplemental Figure 4A). Furthermore, co-depletion of CDKN1A 24 hours after transfection with PAF1 siRNA completely restored the number of G1 phase cells to control numbers after AZD1775 treatment in SETD2 CRISPR KO U20S cells (Figure 4A, Supplemental Figure 4A). Notably, the low intensity of the BrdU staining in the co-depleted cells (siPAF1 + siCDKN1A), including an increase in the BrdU-negative population, suggested that even though cells depleted for both PAF1 and CDKN1A entered S-phase, replication was 
still compromised (Figure 4A). These results indicate that p21 is required for the cell cycle effects observed following PAF1 depletion.

To test whether these p21-induced cell cycle effects were indeed responsible for the PAF1mediated resistance to AZD1775, we tested whether siPAF1-treated SETD2 KO U2OS cells could be re-sensitized to WEE1i through inhibition of $\mathrm{p} 21$. To this goal, we performed cell cycle analyses and viability assays on SETD2 CRISPR KO U2OS transfected with nontargeting control siRNA or PAF1 siRNA using AZD1775 and a pharmacological inhibitor of p21, UC2288, which was shown to downregulate p21 on both the transcriptional and posttranscriptional level (Wettersten et al., 2013) (Figure 4B). Indeed, we found that siPAF1-treated cells with high p21 levels could be killed using high concentrations $(5 \mu \mathrm{M})$ of the p21 inhibitor alone, or with a combination of low doses of AZD1775 and UC2288 (Figure 4B). Next, we reasoned that if loss of PAF1 causes p21 up-regulation in a p53-dependent way, removal of p53 could alleviate p21-mediated G1-arrest in cells depleted of PAF1 components. Therefore, we hypothesized TP53 KO U2OS cells would display a different response to WEE1-inhibitor treatment when depleted of CDC73, CTR9, or PAF1. Indeed, we found that TP53 CRISPR KO U2OS cells depleted for CDC73, CTR9 or PAF1 displayed increased sensitivity to WEE1inhibitor treatment when compared to TP53 WT U2OS cells (Figure 4C), likely a result of dysfunctional p53 and p21 pathway activation, and therefore further increased CDK activity, leading to increased cell death.

To test whether p21 inhibitors could be applied to specifically target SETD2-deficient tumours, we also explored UC2288 sensitivity in SETD2 WT versus SETD2-mutant RCC cells. First, we measured cell viability in SETD2 wild type and SETD2 mutant cells following knockdown of CDKN1A (p21). siRNA treatment significantly reduced p21 protein expression in both cell lines (Figure 4D). As shown in Figure 4E, we found that knockdown of CDKN1A further sensitised RCC cell lines 786-0 and A498 to AZD1775 (Figure 4E) In agreement with our findings in RCC cell lines 786-0 and A498, p21 inhibition caused a small increase in the Sphase population (Supplemental Figure 4B) and further sensitised both SETD2 WT and KO U2OS cells to AZD1775, mostly at lower concentrations, showing p21 has a direct role in determining sensitivity to WEE1-inhibitor treatment (Supplemental Figure 4C). Furthermore, we found that SETD2 KO U2OS cells are more sensitive to p21 inhibition by UC2288 alone when compared to SETD2 WT U2OS cells, suggesting that p21 is a critical vulnerability in high-p21 expressing SETD2 KO cancer cells.

\section{Discussion}

Here, we characterize CDC73, CTR9, and PAF1, components of the PAF1 complex, as important novel regulators of sensitivity to the WEE1 inhibitor AZD1775. We found that loss of CDC73, CTR9, and PAF1 leads to a partial blockage of the G1/S transition, making cells 
less prone to replication catastrophe upon treatment with the WEE1 inhibitor. We propose that in the absence of PAF1, CDC73, or CTR9, elevated p21 as a result of high levels of R-loops promotes cell survival through its role as a cyclin-dependent kinase (CDK) inhibitor under replication stress conditions. p21-dependent CDK inhibition results in the partial blockage of G1/S transition thereby slowing of S-phase progression, and ultimately allowing more time for DNA replication and DNA repair. We also show that PAF1-depleted SETD2-deficient cells can be re-sensitized to AZD1775 through elevation of CDK activity using a p21 inhibitor.

The effect of CDC73, CTR9, or PAF1 depletion on the replication catastrophe phenotype upon WEE1 inhibition was particularly pronounced in SETD2-depleted cells. As elevated CDK activity resulting from Wee1 inactivation is required to manifest the synthetic lethality in between Set2 deletion and Wee1 inactivation in $S$. pombe, we were not surprised to identify loss of function mutations in $c d c 2$ amongst the spontaneous mutants that suppressed the set2 $\Delta$ wee 1-50 interaction (Pai et al., 2019). CDK1 and CDK2 hyperactivation is central to the manifestation of the synthetic lethality between loss of SETD2 and inhibition of WEE1, as elevated CDK activity promotes increased replication origin firing as well as cyclin F-mediated degradation of RRM2, the regulatory subunit of ribonucleotide reductase (RNR) that catalyses the rate limiting step of the formation of deoxyribonucleotides (D'Angiolella et al., 2012; Pfister et al., 2015). This ultimately causes cell death due to dNTP shortage. Considering the well-described role of p21 in regulating CDK activity in mammalian cells and its requirement for G1 arrest, we predict that p21 protects cells from AZD1775-induced $S$ phase damage through a similar, but indirect, modulation of CDK activity. P21 also exists in a complex with the proliferating-cell nuclear antigen (PCNA) (Waga et al., 1994), providing another way through which p53dependent induction of p21 can result in slowing of DNA replication.

Previous studies have shown that ATR inhibition could be rescued with a CDK1 inhibitor that prolonged the cell cycle, thereby providing more time for DNA replication such that mitosis and cell division could complete with more fully replicated DNA (Eykelenboom et al., 2013). Interestingly, though, recent work by Hauge et al., showed that high p21 did not alter Sphase CDK activity but rather the protective effect of p21 in the presence of AZD1775 was mediated through damage reduction, as measured by $\mathrm{pH} 2 \mathrm{AX}$ (Hauge, Macurek and Syljuåsen, 2019). They hypothesised that p21 is involved in restraining replication after WEE1 inhibition, limiting S-phase damage and subsequent cell death. Indeed, this hypothesis aligns with previous work from Gottifredi et al., where it was shown that only cells expressing low p21 immediately progress through the cell cycle upon release from $S$ phase arrest, whereas high p21 cells move much more slowly through the cell cycle, demonstrating that p21 is required for efficient replication restart (Gottifredi et al., 2004)

The direct link between loss of components of the PAF1C and elevated levels of p21 is not yet fully understood, but we propose that high p21 levels following PAF1 depletion are a 
result of increased R-loop formation. PAF1 loss results in reduced $\gamma \mathrm{H} 2 \mathrm{AX}$ and ATM/DNAPK phosphorylation levels and reduced replicative catastrophe in the presence of AZD1775 at 48 hours, suggesting delayed or reduced DNA double-strand break formation, probably as a result of more complete DNA replication. We do, however, observe p53-dependent CDKN1A upregulation between 24 and 48 hours post-AZD1775 treatment in the absence of PAF1, perhaps in an ATR- and Chk1-dependent manner. Therefore, we propose that elevated R-loops in PAF1-depleted cells lead to replication fork slowing (reviewed in Gómez-González \& Aguilera, 2019), alleviating the lethal effects of replication stress in the first S-phase, whereas underreplicated DNA and unrepaired damage, possibly as a result of elevated transcription-replication conflicts, are carried over into the next cell cycle, leading to problems in mitosis and elevated p21 levels in the subsequent G1 phase.

We therefore propose the following model, where loss of components of the PAF1 complex, including CDC73, CTR9 and PAF1, result in up-regulation of p21 through direct PAF1-mediated up-regulation of R-loop formation. High p21 levels are subsequently responsible for 1) slowing down of replication initiation and progression (Hauge et al., 2019; Waga, Hannon, Beach, \& Stillman, 1994) and 2) reduction of CDK activity, leading to a second-cycle G1 arrest, both of which ultimately lead to reduced S-phase damage, rescue of replication catastrophe and increased cellular survival in the presence of AZD1775 (Figure 4F).

Even though PAF1-depleted cells display cross-resistance to other inhibitors of ribonucleotide reductase, including $\mathrm{HU}$ and gemcitabine, they showed reduced cellular survival upon $\gamma$ irradiation, suggesting a complex relationship between the PAF1 complex, nucleotide pools and the response to DNA damage. High levels of p21 in PAF1-depleted cells might, in addition to causing prolonged $\mathrm{G} 1$ arrest in the subsequent cell cycle, also suppress the degradation of RRM2 via inhibition of the CDK2-cyclin E complex, thereby restoring nucleotide pools. In $S$. pombe, previous studies have found that high dNTP levels can lead to increased DNA damage sensitivity and slow progression through S-phase (Fleck et al., 2013; Pai and Kearsey, 2017). A recent study has suggested a role for PAF1 in attenuating radiosensitivity by inhibiting immediate-early response 5 (IER5) transcription, which is involved in cell cycle regulation (Ding et al., 2019; Zheng et al., 2020).

Our results suggest that tumours with high levels of R-loops and concomitant increases in p21 caused by low levels of CDC73, PAF1, and CTR9 would not benefit from treatment with the WEE1 inhibitor. Previous studies have implicated mutations in members of the PAF1C, including CTR9, in tumorigenesis (Hanks et al., 2014). As AZD1775 is currently being investigated in more than fifty clinical trials for cancer treatment, as monotherapy or in combination with chemotherapeutic drugs and/or radiation (www.clinicaltrials.gov), expression levels and mutation status of PAF1C components should be taken into consideration in the clinical context. Finally, we propose that a combination of low doses of AZD1775 (WEE1i) 
and UC2288 (p21i) could be used to improve the therapeutic response of SETD2-deficient cells, even in the context of PAF1 loss, which should be further explored in in vivo studies.

\section{Materials and Methods}

Yeast strains and media. Strains were cultured and stored as described in Moreno, Klar, \& Nurse, 1991. YE6S medium contained 3\% glucose, 0.5\% EZmixTM yeast extract (Sigma), and $1.125 \mathrm{~g} / \mathrm{L}$ supplements mix containing equal amounts of adenine, arginine, L-histidine, Lleucine, lysine, tryptophan, and uracil. Strains used in this study can be found in Table 1.

\begin{tabular}{|c|c|c|}
\hline Strain & Label & Genotype \\
\hline TH344 & wee1-50 h- & wee1-50 h- \\
\hline TH345 & wee1-50 h+ & wee $1-50 \mathrm{ht}$ \\
\hline TH665 & paf1 $1 \Delta$ & $\begin{array}{l}\text { paf1::G418 ade6 - M210. } \\
\text { leu1-32, ura4-D18, his3-D1, } \\
\text { arg3-D4 }\end{array}$ \\
\hline TH2093 & wild type & $\begin{array}{l}\arg 3-D 4, \text { ade6-D1, ura4- } \\
D 18, \text { leu1-32, his3-D1 } \\
h+\end{array}$ \\
\hline TH6236 & $\operatorname{set} 2 \Delta$ & $\begin{array}{l}\text { set2::kanMX ade6-210 leu1- } \\
32 \text { ura4-D18 }\end{array}$ \\
\hline TH7121 & set2 2 wee1-50 & $\begin{array}{l}\text { set2::kanMX wee1-50, ade6- } \\
D 1, \text { ura4-D18, leu1-32, his3- } \\
D 1\end{array}$ \\
\hline TH7122 & set2 $\Delta$ wee $1-50$ & $\begin{array}{l}\text { set2::kanMX wee1-50 arg3- } \\
D 4, \text { ade6-D1, ura4-D18, } \\
\text { leu1-32, his3-D1 }\end{array}$ \\
\hline
\end{tabular}

Serial Dilution Assay. A dilution series for the indicated mutant cells was spotted onto YES plates. Plates were incubated at $25^{\circ} \mathrm{C}, 32^{\circ} \mathrm{C}$, or $36^{\circ} \mathrm{C}$ for $2-3$ days before analysis.

Cell culture. U2OS (human osteosarcoma) and the human glioblastoma cell line T98G cells were obtained from ATCC (ATCC number HTB-96 and CRL-1690). The U2OS SETD2 CRISPR knockout (KO) cell line was generated using the CRISPR-Cas9 technology as previously described (Pfister et al., 2015). The target sequence of the chimeric gRNA scaffold was ACTCTGATCGTCGCTACCAT (first exon of SETD2) (Pfister et al., 2015). The U2OS P53 CRISPR KO cell line was obtained from Dr. Kamila Burdova (V. D'Angiolella Lab). Human renal cell carcinoma cell lines 786-O (SETD2 wild-type) were a kind gift from V. Macaulay and A498 (SETD2 homozygous truncating mutation) cells were obtained from ATCC (ATCC number HTB-44). Human cancer cell lines were cultured in Dulbecco's Modified Eagle's Medium 
(DMEM) supplemented with $10 \% \mathrm{v} / \mathrm{v}$ foetal bovine serum (FBS, Sigma), penicillin (100 units $/ \mathrm{mL}$, Sigma) and streptomycin $\left(0.1 \mathrm{mg} / \mathrm{mL}\right.$, Sigma) at $37^{\circ} \mathrm{C}$ in a humidified atmosphere containing $5 \% \mathrm{CO} 2$.

Transient siRNA-mediated gene knockdown. Cell lines were reverse transfected with short interfering RNAs (siRNAs) (12 nM final concentration) using Lipofectamine RNAiMAX (Invitrogen) according to the manufacturer's instructions. Medium was replaced 16-20 hours after transfection. The target sequences of the siRNAs used in this project are listed below: siCDC73 (ONTARGET plus SMARTpool, 015184), siCDKN1A (ON-TARGET plus SMARTpool, 003471), siCTR9 (ON-TARGET plus SMARTpool, 032246), siLEO1 (ON-TARGET plus SMARTpool, 016579), siPAF1 (ON-TARGET plus SMARTpool, 020349), siRTF1 (ON-TARGET plus SMARTpool, 014104), and siSETD2\#3 (si\#3) (Dharmacon): GAAACCGUCUCCAGUCUGU, and siSETD2\#5 (si\#5) (Dharmacon): UAAAGGAGGUAUAUCGAAU.

Drug treatment. All inhibitors were dissolved in Dimethyl sulfoxide (DMSO) and stored at $80{ }^{\circ} \mathrm{C}$ per the manufacturer's instructions. Inhibitors used in this study are AZD1775 (MK1775) (Axon Medchem) and Gemcitabine (Sigma Aldrich).

Clonogenic Survival Assays. Cells were serially diluted into 6-well plates and allowed to attach for 24 hours. They were then exposed to ionising radiation (IR) and grown in fresh medium for 10 days. Colonies were stained with crystal violet and manually counted. Clonogenic survival was expressed as $\%$ colonies relative to untreated cells.

Quantitative RT-PCR (Reverse Transcription-Polymerase Chain Reaction). Total RNA was purified from cell pellets with the RNeasy mini kit (Qiagen). RNA concentration was determined using a Nanodrop 1000 spectrophotometer (Thermo Scientific). Purified total RNA (1 $\mu \mathrm{g}$ ) was reverse transcribed using the superscript iii first strand synthesis supermix for qRT PCR kit (Invitrogen), according to the manufacturer's instructions. Quantitative RT- PCR was performed using Absolute Blue QPCR SYBR green, low ROX Mix (Thermo Scientific). The comparative CT method was applied for quantification of gene expression. Primers are available upon request.

Resazurin assay for cell viability. 48 hours following drug treatment, culture medium was removed and fresh medium containing $10 \mu \mathrm{g} / \mathrm{mL}$ resazurin was added to each well. The fluorescent signal was measured by a fluorescence plate reader (BMG Labtech POLARstar OMEGA) after 2 hours of incubation at $37^{\circ} \mathrm{C}$.

Fluorescence-activated cell sorting (FACS) analysis. Cells were collected by trypsinization and fixed in $70 \%$ ice-cold ethanol (EtOH). Fixed cells were resuspended in $1 \mathrm{~mL}$ PBS supplemented with $10 \mu \mathrm{L} 1 \mathrm{mg} / \mathrm{mL}$ propodium iodide $(\mathrm{PI})$. Cells were incubated for $>30 \mathrm{~min}$ 
at RT and then analysed for DNA content by flow cytometry. For simultaneous staining of $\mathrm{gH} 2 \mathrm{AX}$ and EdU, cells were labelled with $\mathrm{gH} 2 \mathrm{AX}$ as and EdU as previously described (HB et al., 2020). Briefly, cells were incubated for $1 \mathrm{~h}$ with $2 \mu \mathrm{M}$ EdU (Thermo Fisher) and fixed in $70 \%$ ethanol, prior to labelling with primary ( $\mathrm{gH} 2 \mathrm{AX}$ antibodies (clone JBW301, Millipore)) and secondary (anti-mouse Alexa488, A21202 life technologies) antibodies. EdU was detected using the Click-iT Plus EdU Alexa Fluor 594 Flow Cytometry Assay Kit (Thermo Fisher). For the experiments in Figure 2C-D, a barcoded (with Alexa Fluor 647) control sample of nontreated cells was added to all the individual samples, to eliminate sample to sample variation during staining. Samples were analysed in a LSRII flow cytometer (BD Biosciences) and processed in FACSDiva and FlowJo software (Both BD Biosciences).

Immunoblotting. Cells were lysed in lysis buffer $(50 \mathrm{mM}$ Tris $\mathrm{HCl} \mathrm{pH} \mathrm{7.5,} 150 \mathrm{mM} \mathrm{NaCl}$, $1 \%$ Triton) supplemented with 1:50 fresh protease inhibitor cocktail and 1:100 fresh phosphatase inhibitor cocktail (1mM NaOVa; $10 \mathrm{mM} \mathrm{NaF} ; 10 \mathrm{mM} \mathrm{NaPyrophosphate)} \mathrm{and}$ immunoblotting was performed as described previously. Targets of the primary antibodies used for western blotting were as follows: Apoptosis Cocktail (pro/p17-caspase-3, cleaved caspase-3, cleaved-PARP, cleaved-actin, 1:500, Abcam), RRM2, p21 (1:1000, Cell Signalling), p-CDK(S), p-ATR(S428), ATR, p-Chk1(S317), Chk1, P-ATM S1981 (Cell signalling). DNA-PK pS2056 (abcam), p-RPA S4S8 (Bethyl), CDK1 (sc-54 Santa Cruz biotech), pCDK1 Y15 (Cell signalling), PAF1 (Cell Signaling), Lamin $B$ (cell signalling), GAPDH and Tubulin. Stain free technology (Biorad) was used for total protein detection in Figure 2E.

Slot Blot. U2OS cells were lysed in $200 \mathrm{uL}$ lysis buffer $(100 \mathrm{mM}$ Tris- $\mathrm{HCl}[\mathrm{pH} 8.5], 5 \mathrm{mM}$ EDTA, $0.2 \% \mathrm{SDS}$, and $100 \mathrm{mM} \mathrm{NaCl}$ ) containing $0.5 \mathrm{mg} / \mathrm{ml}$ proteinase $\mathrm{K}$ and left at $55^{\circ} \mathrm{C}$, $350 \mathrm{RPM}$ O/N. Genomic DNA was precipitated with isopropanol for $5 \mathrm{~min}$ RT, spun down for $3 \mathrm{~min}$ at $3000 \mathrm{~g}\left(4^{\circ} \mathrm{C}\right.$,) washed with $500 \mu \mathrm{L} 70 \%$ ethanol and spun down at for $3 \mathrm{~min}$ at $3000 \mathrm{~g}\left(4^{\circ} \mathrm{C},\right)$. Supernatant was removed and samples were air-dried. Pellets were resuspended in 100 - $200 \mathrm{uL}$ Tris-EDTA (TE) buffer. gDNA was mixed well and left at $55^{\circ} \mathrm{C}$ for $10 \mathrm{~min}$ to dissolve. All samples were diluted to give $50 \mu \mathrm{g}$ gDNA in a volume of 100 $\mu \mathrm{L}$ TE. 2uL RNase A (10 mg/mL, Thermo Cat\# EN0531) was added to each and incubated $2 \mathrm{hr} 37^{\circ} \mathrm{C}, 300 \mathrm{RPM}$. For the RNaseH1 control, $20 \mu \mathrm{L}$ gDNA was added to $25 \mu \mathrm{L}$ TE + $5 \mathrm{uL}$ of $10 \mathrm{X}$ RNaseH1 buffer $+2 \mathrm{uL} \mathrm{RNaseH} 1$ and incubated $\mathrm{O} / \mathrm{N} 37^{\circ} \mathrm{C}, 300 \mathrm{RPM}$. The remaining untreated gDNA was also incubated $\mathrm{O} / \mathrm{N} 37^{\circ} \mathrm{C}, 300 \mathrm{RPM}$. The gDNA samples (5 $\mu \mathrm{g}$ for 59.6 blot and $0.5 \mu \mathrm{g}$ for ssDNA blot) were blotted onto a positively charged nylon transfer membrane (GE Healthcare Cat\# RPN203B) using a slot blot apparatus (Bio-Rad). ssDNA blot was incubated in denaturing buffer $(0.4 \mathrm{M} \mathrm{NaOH}$ and $0.6 \mathrm{M} \mathrm{NaCl})$, shaking for $10 \mathrm{~min} \mathrm{RT}$, followed by neutralizing buffer $(1.5 \mathrm{M} \mathrm{NaCl}$ and $0.5 \mathrm{M}$ Tris $[\mathrm{pH}$ 7.4]) shaking for $10 \mathrm{~min}$ RT. Blots were quickly dried using wattman paper and UV crosslinked using $(0.12$ 
$\mathrm{J} / \mathrm{m} 2$ ). Blots were probed $\mathrm{O} / \mathrm{N}$ using S9.6 (1:1000) or ssDNA-specific antibodies (1:5000), washed in TBS-T, secondary antibody (Anti-Ms-HRP, 1:5000, 2hr RT) and imaged.

Statistical analysis. Unless stated otherwise, all data in the figures and corresponding text are expressed as the mean $+/$ - SEM of $n$ samples $(n \geq 3$ ). Student's unpaired t-tests were used for comparisons between two or more experimental groups, without assuming a consistent SD. P-values of $<0.05$ were considered statistically significant. 


\section{References}

Abbas, T. and Dutta, A. (2009) 'p21 in cancer: intricate networks and multiple activities.', Nature reviews. Cancer, 9(6), pp. 400-14. doi: 10.1038/nrc2657.

Alvino, G. M. et al. (2007) 'Replication in Hydroxyurea: It's a Matter of Time', Molecular and Cellular Biology. doi: 10.1128/MCB.00719-07.

van Bijsterveldt, L. et al. (2020) 'The challenge of combining chemo- and radiotherapy with checkpoint kinase inhibitors', Clinical Cancer Research, p. clincanres.3358.2020. doi: 10.1158/1078-0432.CCR-20-3358.

Cerqueira, N. M. F. S. A., Fernandes, P. A. and Ramos, M. J. (2007) 'Understanding ribonucleotide reductase inactivation by gemcitabine', Chemistry - A European Journal. doi: 10.1002/chem.200700260.

Chen, F. X. et al. (2015) 'PAF1, a Molecular Regulator of Promoter-Proximal Pausing by RNA Polymerase II', Cell, 162(5), pp. 1003-1015. doi: 10.1016/j.cell.2015.07.042.

Crossley, M. P., Bocek, M. and Cimprich, K. A. (2019) 'R-Loops as Cellular Regulators and Genomic Threats', Molecular Cell. doi: 10.1016/j.molcel.2019.01.024.

D'Angiolella, V. et al. (2012) 'Cyclin F-mediated degradation of ribonucleotide reductase M2 controls genome integrity and DNA repair', Cell. Elsevier Inc., 149(5), pp. 1023-1034. doi: 10.1016/j.cell.2012.03.043.

Ding, K. K. et al. (2019) 'Overexpression of the immediate early response 5 gene increases the radiosensitivity of HeLa cells', Oncology Letters. doi: 10.3892/ol.2019.10590.

Edmunds, J. W., Mahadevan, L. C. and Clayton, A. L. (2008) 'Dynamic histone H3 methylation during gene induction: HYPB/Setd2 mediates all H3K36 trimethylation', The EMBO Journal, 27, pp. 406-420. doi: 10.1038/.

Eykelenboom, J. K. et al. (2013) 'ATR Activates the S-M Checkpoint during Unperturbed Growth to Ensure Sufficient Replication Prior to Mitotic Onset', Cell Reports. doi: 10.1016/j.celrep.2013.10.027.

Fleck, O. et al. (2013) 'Spd1 accumulation causes genome instability independently of ribonucleotide reductase activity but functions to protect the genome when deoxynucleotide pools are elevated', Journal of Cell Science. doi: 10.1242/jcs.132837.

Gómez-González, B. and Aguilera, A. (2019) 'Transcription-mediated replication hindrance: A major driver of genome instability', Genes and Development. Cold Spring Harbor Laboratory Press, pp. 1008-1026. doi: 10.1101/gad.324517.119.

Gottifredi, V. et al. (2004) 'Decreased p21 Levels Are Required for Efficient Restart of DNA Synthesis after S Phase Block', Journal of Biological Chemistry. doi: 10.1074/jbc.M310373200.

Hanahan, D. and Weinberg, R. A. (2011) 'Hallmarks of cancer: The next generation', Cell, pp. 646-674. doi: 10.1016/j.cell.2011.02.013.

Hanks, S. et al. (2014) 'Germline mutations in the PAF1 complex gene CTR9 predispose to Wilms tumour', Nature Communications. doi: 10.1038/ncomms5398.

Hauge, S., Macurek, L. and Syljuåsen, R. G. (2019) 'p21 limits S phase DNA damage caused by the Wee1 inhibitor MK1775', Cell Cycle. doi: 10.1080/15384101.2019.1593649. HB, L. et al. (2020) 'WDR82/PNUTS-PP1 Prevents Transcription-Replication Conflicts by Promoting RNA Polymerase II Degradation on Chromatin', Cell reports. Cell Rep, 33(9). doi: 10.1016/J.CELREP.2020.108469. 
Krogan, Nevan J et al. (2003) 'Methylation of histone H3 by Set2 in Saccharomyces cerevisiae is linked to transcriptional elongation by RNA polymerase II.', Molecular and cellular biology, 23(12), pp. 4207-18. doi: 10.1128/MCB.23.12.4207.

Krogan, Nevan J. et al. (2003) 'The Paf1 complex is required for histone H3 methylation by COMPASS and Dot1p: Linking transcriptional elongation to histone methylation', Molecular Cell. doi: 10.1016/S1097-2765(03)00091-1.

Liaw, H., Lee, D. and Myung, K. (2011) 'DNA-PK-dependent RPA2 hyperphosphorylation facilitates DNA repair and suppresses sister chromatid exchange', PLOS ONE. PLoS One, 6(6). doi: 10.1371/journal.pone.0021424.

Matheson, C. J., Backos, D. S. and Reigan, P. (2016) 'Targeting WEE1 Kinase in Cancer', Trends in Pharmacological Sciences, pp. 872-881. doi: 10.1016/j.tips.2016.06.006.

Matos, D. A. et al. (2020) 'ATR Protects the Genome against R Loops through a MUS81Triggered Feedback Loop', Molecular Cell. doi: 10.1016/j.molcel.2019.10.010.

Mbogning, J. et al. (2013) 'The PAF Complex and Prf1/Rtf1 Delineate Distinct Cdk9Dependent Pathways Regulating Transcription Elongation in Fission Yeast', PLoS Genetics. doi: $10.1371 /$ journal.pgen.1004029.

$\mathrm{Ng}, \mathrm{H}$. H. et al. (2003) 'Targeted recruitment of Set1 histone methylase by elongating Pol II provides a localized mark and memory of recent transcriptional activity', Molecular Cell. doi: 10.1016/S1097-2765(03)00092-3.

Pai, C. C. et al. (2019) 'An essential role for dNTP homeostasis following CDK-induced replication stress', Journal of Cell Science. doi: 10.1242/jcs.226969.

Pai, C. C. and Kearsey, S. E. (2017) 'A critical balance: DNTPs and the maintenance of genome stability', Genes. doi: 10.3390/genes8020057.

Parker, L. L. and Piwnica-Worms, H. (1992) 'Inactivation of the p34cdc2-Cyclin B Complex by the Human WEE1 Tyrosine Kinase', Science, 257(1989), pp. 1955-1957. doi:

10.1126/science.1384126.

Pfister, S. X. et al. (2015) 'Inhibiting WEE1 Selectively Kills Histone H3K36me3-Deficient Cancers by dNTP Starvation', Cancer Cell. Elsevier Ltd, 28(5), pp. 557-568. doi: 10.1016/j.ccell.2015.09.015.

Poli, J. et al. (2012) 'dNTP pools determine fork progression and origin usage under replication stress', The EMBO Journal, 31(4), pp. 883-894. doi: 10.1038/emboj.2011.470. Rorà, A. G. L. di et al. (2020) 'A WEE1 family business: regulation of mitosis, cancer progression, and therapeutic target', Journal of Hematology \& Oncology. BioMed Central, 13(1). doi: 10.1186/S13045-020-00959-2.

Russell, P. and Nurse, P. (1987) 'Negative regulation of mitosis by wee1+, a gene encoding a protein kinase homolog', Cell. Elsevier, 49(4), pp. 559-567. doi: 10.1016/00928674(87)90458-2.

Shechter, D., Costanzo, V. and Gautier, J. (2004) 'ATR and ATM regulate the timing of DNA replication origin firing', Nature Cell Biology, 6(7), pp. 648-655. doi: 10.1038/ncb1145.

Shivji, M. K. K. et al. (2018) 'BRCA2 Regulates Transcription Elongation by RNA Polymerase II to Prevent R-Loop Accumulation', Cell Reports, 22(4), pp. 1031-1039. doi: 10.1016/j.celrep.2017.12.086.

Vos, S. M. et al. (2018) 'Structure of activated transcription complex Pol II-DSIF-PAF-SPT6', Nature. Springer US. doi: 10.1038/s41586-018-0440-4.

Vos, S. M. et al. (2020) 'Structure of complete Pol II-DSIF-PAF-SPT6 transcription complex reveals RTF1 allosteric activation', Nature Structural \& Molecular Biology. doi: 
10.1038/s41594-020-0437-1.

Waga, S. et al. (1994) 'The p21 inhibitor of cyclin-dependent kinases controls DNA replication by interaction with PCNA', Nature. doi: 10.1038/369574a0.

Wahba, L. et al. (2011) 'RNase H and Multiple RNA Biogenesis Factors Cooperate to Prevent RNA:DNA Hybrids from Generating Genome Instability', Molecular Cell. doi: 10.1016/j.molcel.2011.10.017.

Wettersten, H. I. et al. (2013) 'A novel p21 attenuator which is structurally related to sorafenib', Cancer Biology and Therapy. Cancer Biol Ther, 14(3), pp. 278-285. doi: $10.4161 /$ cbt.23374.

Wood, A. et al. (2003) 'The Paf1 complex is essential for histone monoubiquitination by the Rad6-Bre1 complex, which signals for histone methylation by COMPASS and Dot1p', Journal of Biological Chemistry. doi: 10.1074/jbc.C300269200.

$\mathrm{Yu}, \mathrm{M}$. et al. (2015) 'RNA polymerase II-associated factor 1 regulates the release and phosphorylation of paused RNA polymerase II', Science. doi: 10.1126/science.aad2338.

Zheng, J. J. et al. (2020) 'Novel role of PAF1 in attenuating radiosensitivity in cervical cancer by inhibiting IER5 transcription', Radiation Oncology. doi: 10.1186/s13014-020-01580-w.

\section{Figure Legends}

Figure 1. PAF1C-mediated resistance to depleted dNTP pools. (A) The growth response of wild-type $S$. pombe, set2 $\Delta$, wee1-50, paf1 $\Delta$, set2 $\Delta$ wee1-50, set2 $\Delta$ paf1 $\Delta$, and set2 $\Delta$ wee150 paf1 $\Delta$ was compared by spotting 5 -fold serial dilutions of each strain onto YE6S medium. Plates were the incubated for an appropriate time at either 25,32 or $35.5{ }^{\circ} \mathrm{C}$. (B-C) SETD2 WT and SETD2 CRISPR KO U2OS cells after $72 \mathrm{~h}$ exposure to different concentrations of AZD1775 (75, 150, 300 and $600 \mathrm{nM})$. (D-E) Viability assay to examine the impact of other PAF1 complex factors (including CDC73 and CTR9) silencing on AZD1775-sensitivity. Survival fraction of non-targeting control, CDC73, and CTR9 siRNA treated SETD2 WT and SETD2 CRISPR KO cells after $72 \mathrm{~h}$ exposure to different concentrations of AZD1775 (75, 150, 300 and $600 \mathrm{nM}$ ). Data points and bars represent the mean and SEM of $\geq$ three independent experiments; * $\mathrm{P}<0.05$; ${ }^{* *} \mathrm{P}<0.01$; and ${ }^{* * *} \mathrm{P}<0.001$. (F) Viability assay to examine the impact of PAF1 silencing on $\mathrm{HU}$ or gemcitabine. Survival fraction of non-targeting control and PAF1 siRNA treated SETD2 WT and SETD2 CRISPR KO U2OS cells after $72 \mathrm{~h}$ exposure to different concentrations of $\mathrm{HU}(150,300,600$ and $1200 \mathrm{uM})$ or gemcitabine $(12.5,25,50$ or $100 \mathrm{nM})$. Data points and bars represent the mean and SEM of $\geq$ three independent experiments; * $\mathrm{P}<0.05$; ${ }^{* *} \mathrm{P}<0.01$; and ${ }^{* * *} \mathrm{P}<0.001$. (G) Viability assay to examine the sensitivity to $\mathrm{HU}$ in gene deletions of PAF1C components in S. pombe.

Figure 2 - Loss of PAF1 results in G1 accumulation and decreased S-phase associated DNA damage. (A) 48 hours following DMSO or AZD1775-treatment (300 nM), control or 
siPAF1 treated SETD2 KO U2OS cells were pulse-labelled with BrdU for $30 \mathrm{~min}$ and collected for cell cycle analysis. The data shown are from a single representative experiment out of three repeats. Numbers are the relative percentage of cell cycle stage. (B) Quantification of data shown in (A). (C) SETD2 CRISPR KO U2OS were treated with control or siPAF1 siRNA and exposed to $250 \mathrm{nM}$ of AZD1775 for 48 hours. One representative experiment showing $\mathrm{yH} 2 \mathrm{AX}$ vs DNA (left panel) and EdU vs DNA (right panel). The numbers indicate the percentages of cells in the high $\mathrm{yH} 2 \mathrm{AX}$-gated population (shown in black box). These cells correspond with the non-replicating S-phase cells as shown in the EdU plots (blue population). (D) Quantification of the $\mathrm{yH} 2 \mathrm{AX}$ medium and high populations shown in (C). (E) Western blot analysis of SETD2 CRISPR KO U2OS cells exposed to either non-targeting control siRNA or PAF1 siRNA and/or treated with either DMSO or $300 \mathrm{nM}$ AZD1775. Lamin B was used as a loading control. (F) $48 \mathrm{hr}$ following AZD1775-treatment, control, siCDC73, or siCTR9 treated SETD2 KO U2OS cells were pulse-labelled with BrdU for $30 \mathrm{~min}$ and collected for cell cycle analysis.

Figure 3. Loss of PAF1 causes R-loop dependent up-regulation of p21 in SETD2-deficient cells. (A) Western blot analysis of p21 protein levels in SETD2 WT and SETD2 CRISPR KO U2OS cells after 48 hours of treatment with either non-targeting control siRNA or PAF1 siRNA. Tubulin was used as a loading control. (B) qRT-PCR analysis of CDKN1A levels normalised to $18 \mathrm{~S}$ in U2OS cells transfected with the indicated siRNAs $(n=3)$. (C) Western Blot analysis of $\mathrm{p}-\mathrm{CDK}(\mathrm{S})$ activity following siPAF1 with and without AZD1775 in SETD2 WT U2OS cells. (D) RNA:DNA hybrid slot blot of genomic DNA \pm RNaseH1 treatment from cells treated with siNT, siSETD2, siPAF1 or combined siSETD2 and siPAF1. (E) Western blot analysis of p21 protein levels in SETD2 KO U2OS cells. Immunoblot staining for the V5epitope confirms RNAseH1 overexpression.

Figure 4. Paf1-mediated suppression is dependent on the p53/p21 axis. (A) $48 \mathrm{hr}$ following DMSO -or AZD1775-treatment, siPAF1, or siCDKN1A + siPAF1 treated SETD2 KO U2OS cells. The data shown are from a single representative experiment out of three repeats. Numbers are the relative percentage of cell cycle stage. (B) SETD2 CRISPR KO U2OS cells treated with siNT or siPAF1 after 48h exposure to different concentrations of AZD1775 (0-10 $\boldsymbol{\mu M}$ ) and UCC2288 (p21 inhibitor). Survival fraction of non-targeting control, CDC73, and CTR9 (C) siRNA treated TP53 CRISPR KO cells after $72 \mathrm{~h}$ exposure to different concentrations of AZD1775 (75, 150, 300 and $600 \mathrm{nM})$. Data points and bars represent the mean and SEM

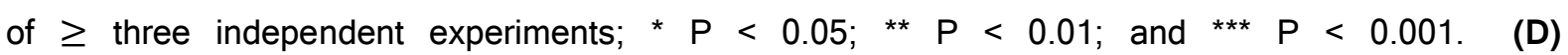
Western blot analysis of p21 protein levels in SETD2 WT and SETD2 CRISPR KO U2OS cells exposed to either non-targeting control siRNA or p21 siRNA. Tubulin was used as a loading control. (E) Cell viability is further reduced when WEE1 inhibition is combined with p21 knock down in SETD2 WT and SETD2 mutant cancer cell lines. (F) Model where loss of CDC73, CTR9 and PAF1 results in resistance to WEE1i treatment. 
Supplemental Figure 1. (A) Validation of PAF1 siRNA knockdown efficiency by Western Blot in SETD2 WT and SETD2 CRISPR KO U2OS cells 72 hours post-siRNA treatment. (B) Viability assay to examine the impact of PAF1 silencing on AZD1775-sensitivity. Survival fraction of non-targeting control and PAF1 siRNA treated (A-B) 786-0 (SETD2 WT) and A498 (SETD2-mutant) renal cancer cells. C) Cleaved PARP, procaspase 3, cleaved caspase 3 and actin protein expression in SETD2 WT and SETD2 CRISPR KO U2OS cells 48 hours posttreatment with either $200 \mathrm{nM}$ DMSO or $200 \mathrm{nM}$ AZD1775 was assessed using Western Blot. D) Validation of CDC73 and CTR9 siRNA knockdown efficiency by Western Blot in SETD2 WT and SETD2 CRISPR KO U2OS cells 72 hours post-siRNA treatment.

Supplemental Figure 2. (A) $\mathrm{yH} 2 \mathrm{AX}$ level measured by flow cytometry after wash-out of MK1775. U20S cells were treated with $1 \mu \mathrm{M}$ MK-1775 for $6 \mathrm{~h}$, then washed with PBS and cultured further for $4 \mathrm{~h}$. Cells were fixed and stained for $y \mathrm{H} 2 \mathrm{AX}$ and DNA content. The strong and intermediate $\mathrm{yH} 2 \mathrm{AX}$ populations were gated and percentages determined. One representative experiment out of 15 experiments using different MK-1775 concentration. (B) The strong $\mathrm{yH} 2 \mathrm{AX}$ population ceases replication. U20S cells were treated with $1 \mu \mathrm{M} \mathrm{MK}-177$ 5 for $7 \mathrm{~h}$, washed in PBS and cultured further for $2 \mathrm{~h}$. For the last hour $1 \mu \mathrm{M}$ EdU was added. Cells were fixed and stained for $y H 2 A X$, EdU and DNA content. The strong $y H 2 A X$ population was gated and is indicated in black in the EdU plot. (C) Quantification of p21, pATM/ATM and PRPA levels from Western Blot shown in Figure 2E.

Supplemental Figure 3. (A) SETD2 WT and (B) SETD2 CRISPR KO U2OS were treated with control, siCDC73, siCTR9 or siPAF1 and subsequently synchronized the $G_{1} / S$ transition by a double thymidine block (DTB) and then released by addition of fresh medium. Cells were incubated with $\mathrm{BrdU}$ for $30 \mathrm{~min}$ and collected for cell cycle analysis 4, 8, and 12 hours after release. (C) Western blot analysis of RRM2 protein levels in SETD2 WT and SETD2 CRISPR KO U2OS cells exposed to either non-targeting control siRNA or PAF1 siRNA and/or treated with either DMSO or $300 \mathrm{nM}$ AZD1775. Tubulin was used as a loading control.

Supplemental Figure 4. A) $48 \mathrm{hr}$ following DMSO -or AZD1775-treatment, control, siCDKN1A, siPAF1, or siCDKN1A + siPAF1 treated SETD2 KO U2OS cells were pulse-labelled with BrdU for $30 \mathrm{~min}$ and collected for cell cycle analysis. The data shown are from a single representative experiment out of three repeats. Numbers are the relative percentage of cell cycle stage. B) $48 \mathrm{hr}$ following DMSO, AZD1775- and/or UC2288 treatment, SETD2 KO U2OS cells were collected for cell cycle analysis. C) SETD2 WT and SETD2 CRISPR KO U2OS cells after $48 \mathrm{~h}$ exposure to different concentrations of AZD1775 (0-10 $\mu \mathrm{M})$ and UCC2288 (p21 inhibitor). 
A)

Figure 1

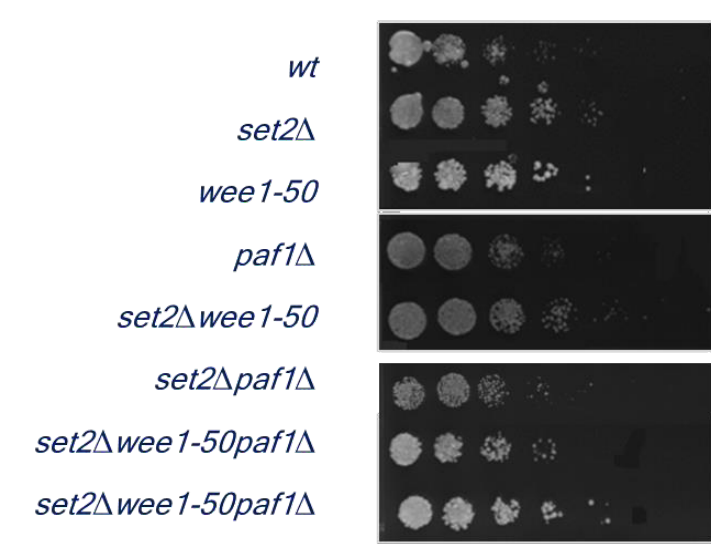

$25{ }^{\circ} \mathrm{C}$
B)

סे

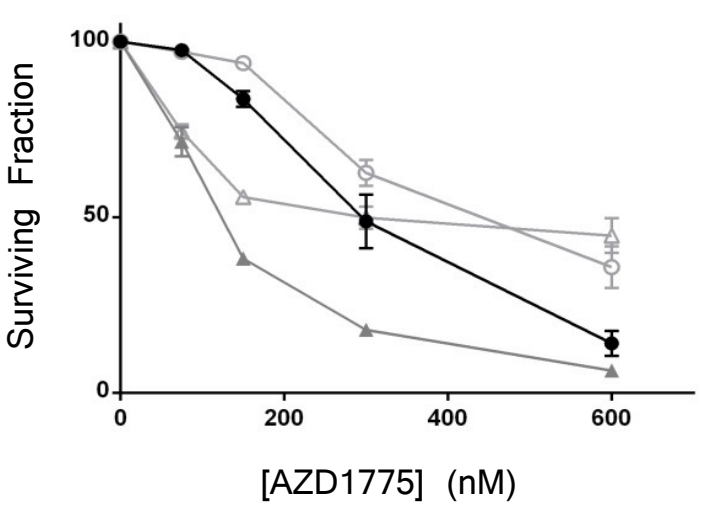

D)

SETD2 WT U2OS

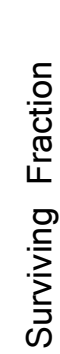

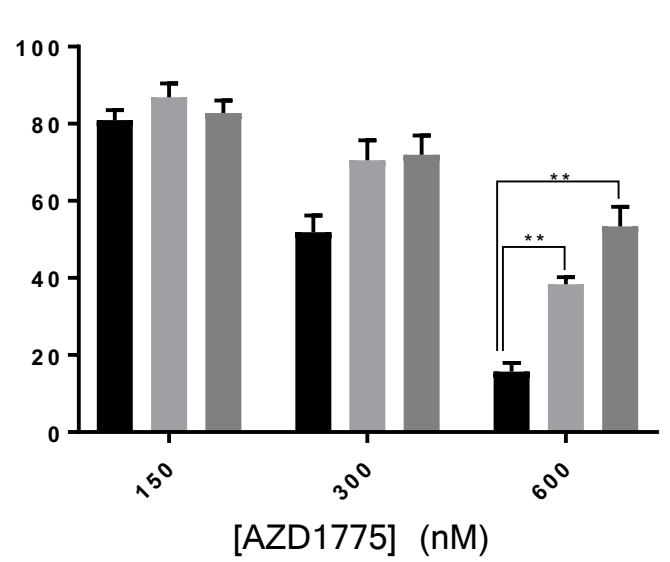

E)

$32{ }^{\circ} \mathrm{C}$

C)

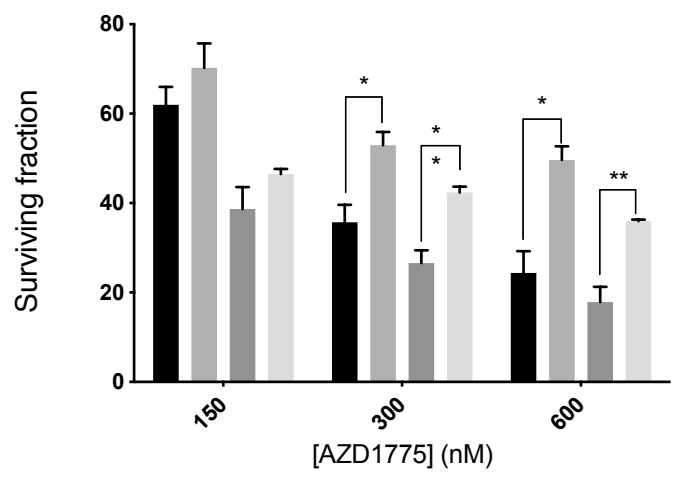

SETD2-WT NT

SETD2-WT SIPAF1

SETD2-MUT NT

SETD2-MUT SIPAF1

SETD2 KO

\# NT

$\triangle$ siPAF1
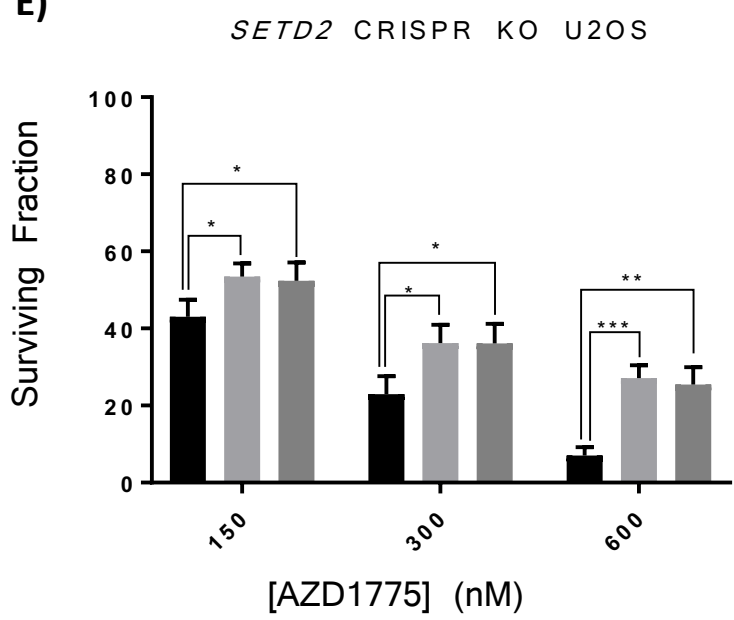

nT

$\operatorname{siCDC} 73$

siCTR 9 
E)

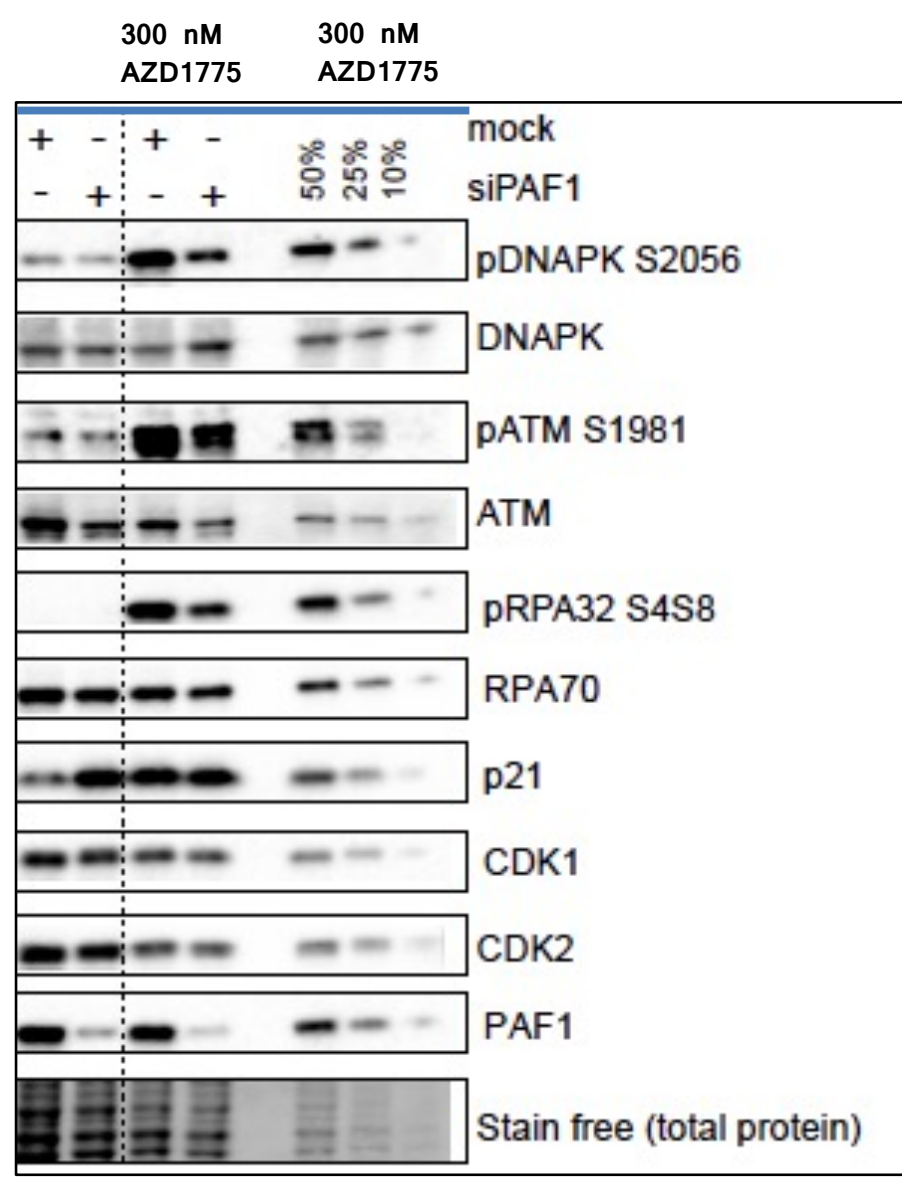

F)
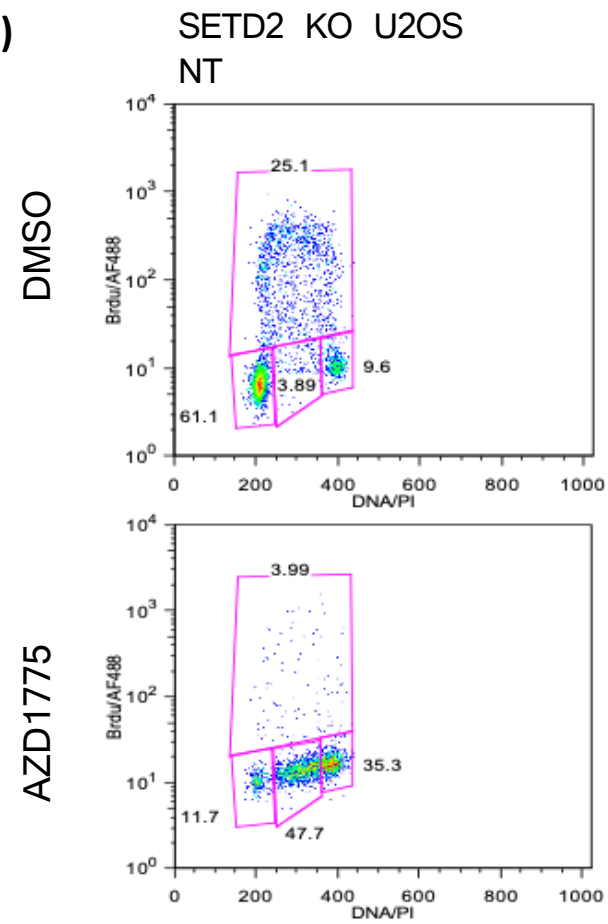

SETD2 KO U2OS

NT
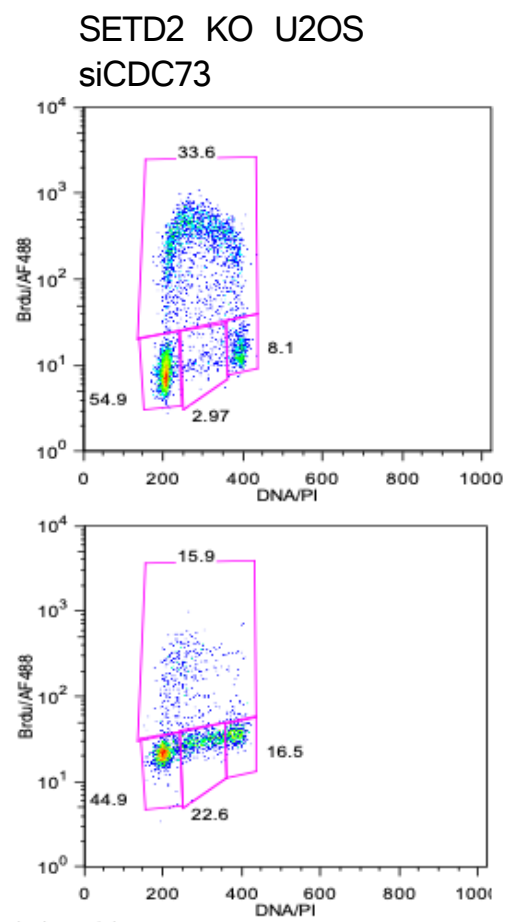

SETD2 KO U2OS SiCDC73
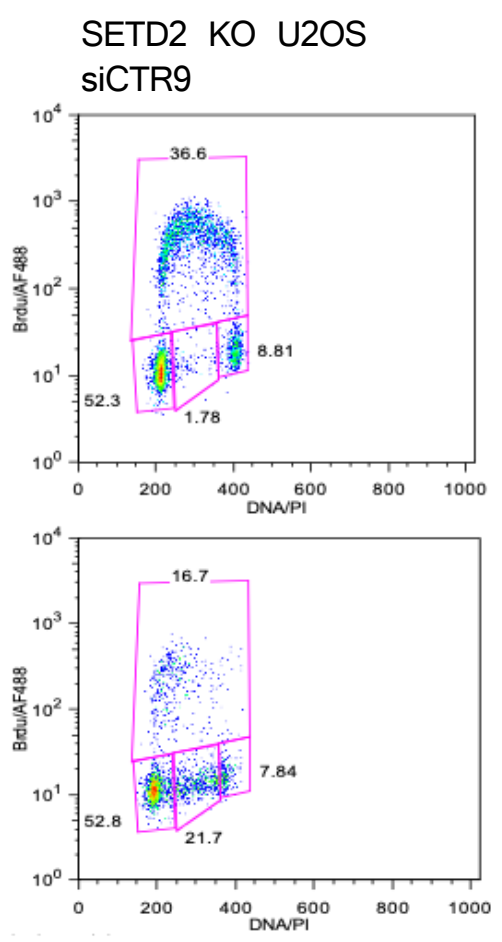

SETD2 KO U2OS siCTR9

Figure 2 
A)

B)
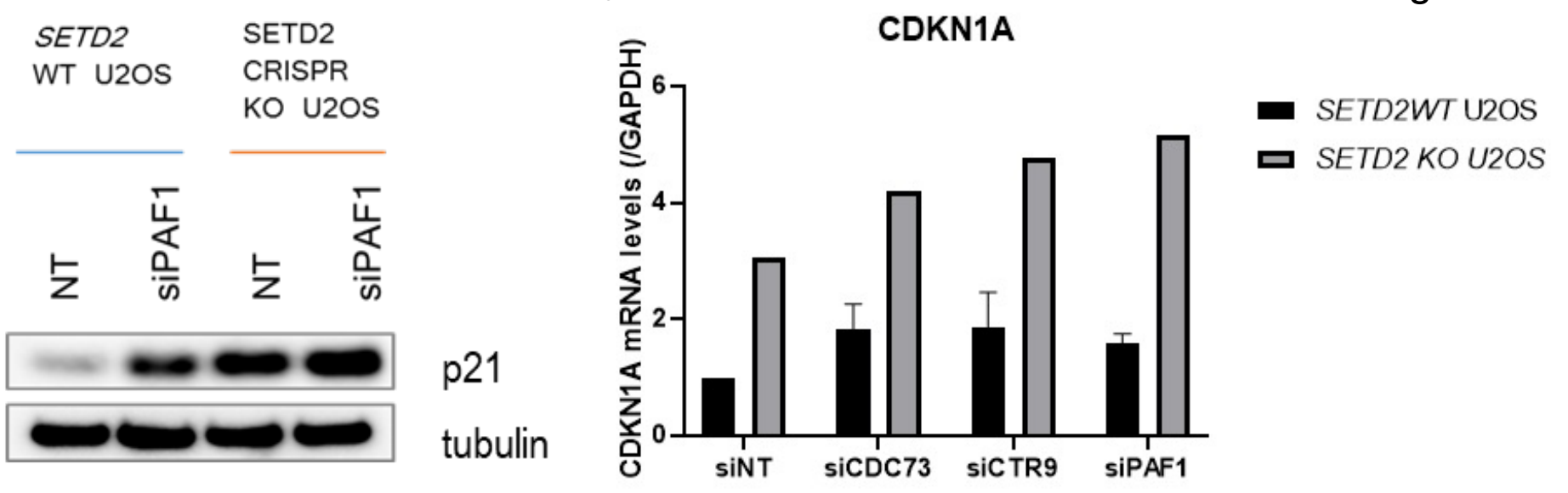

tubulin

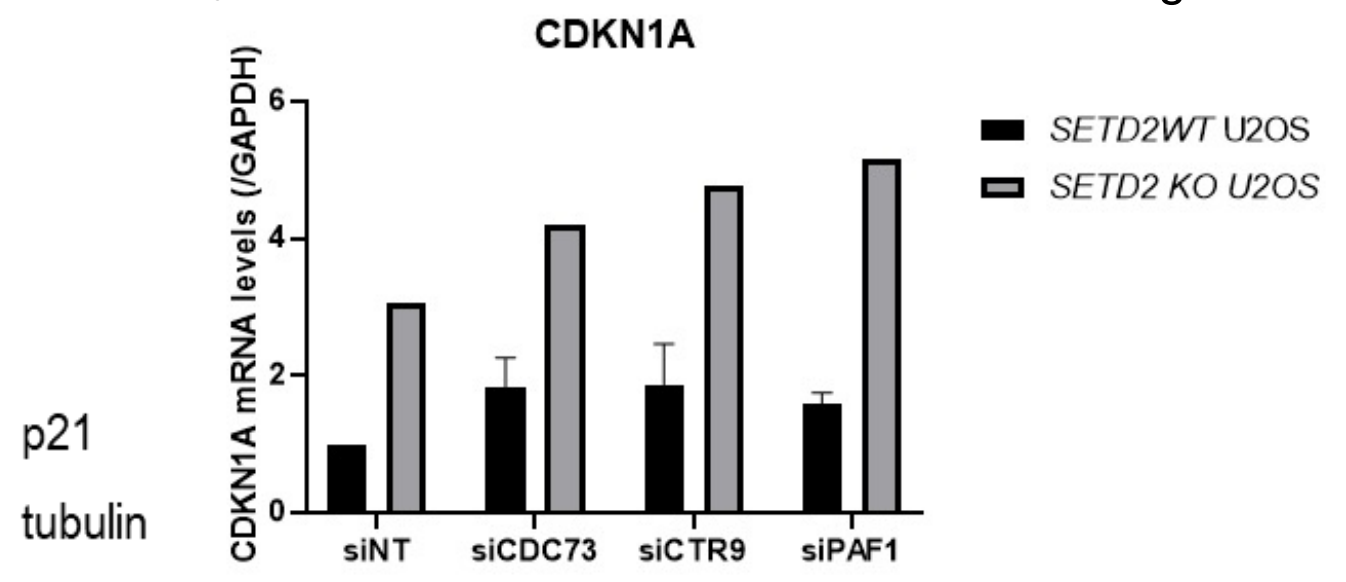

Figure 3

CDK

substrate

phosphorylation

D)

S9.6 ssDNA

E)

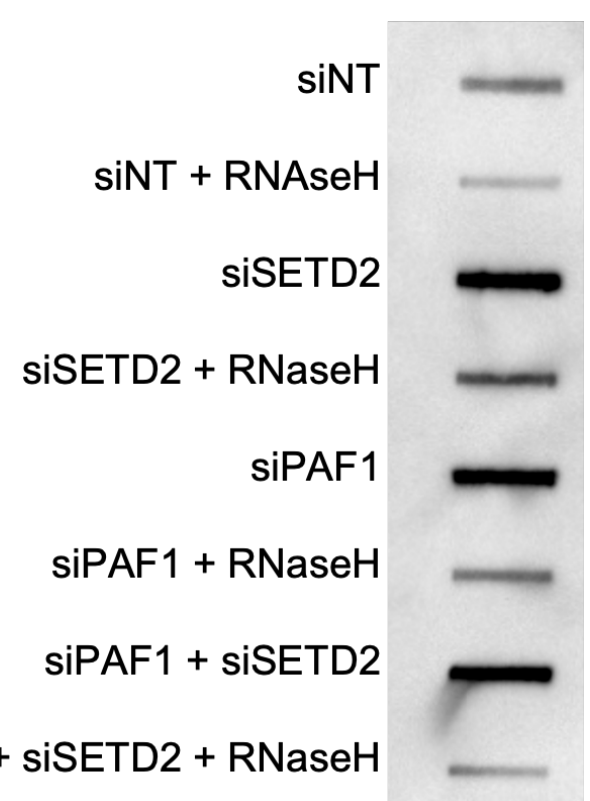

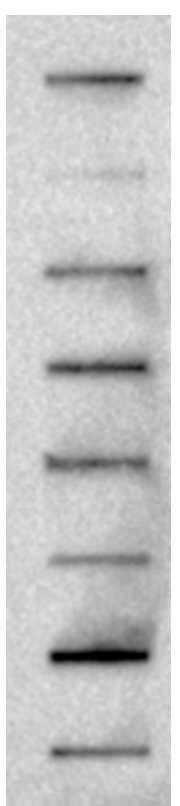

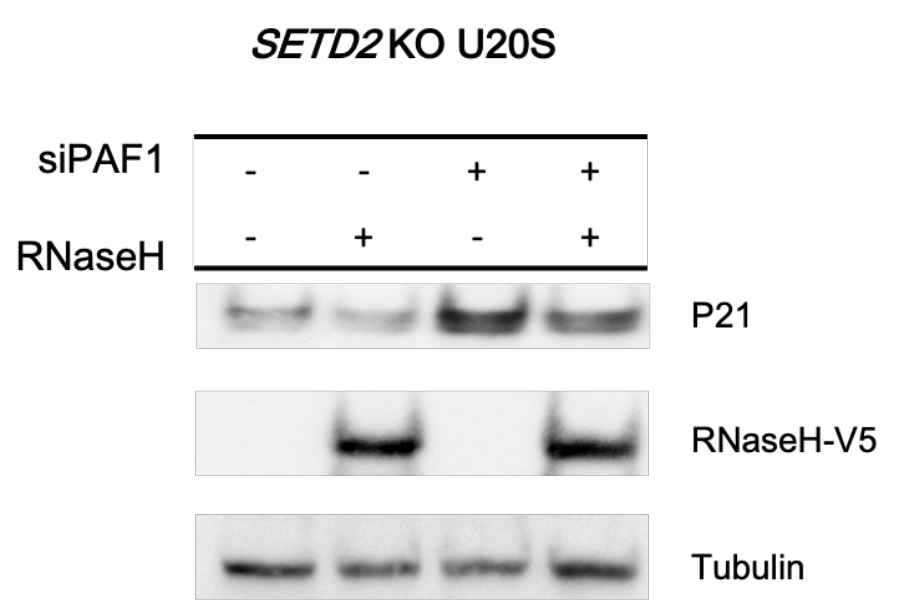

siPAF1 + siSETD2 + RNaseH 
A)

SETD2 CRISPR KO U2OS SIPAF1 DMSO
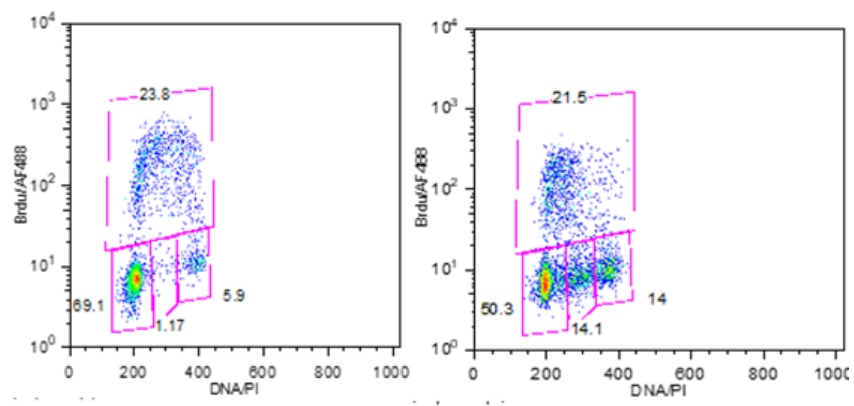

SETD2 CRISPR KO U2OS SiPAF1 AZD1775
SETD2 CRISPR KO U2OS

SiPAF1 + SiCDKN1A AZD1775

Figure 4

B)

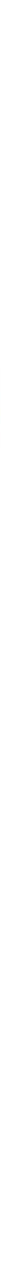

B) 


\section{P53 CRISPR KO U2OS}

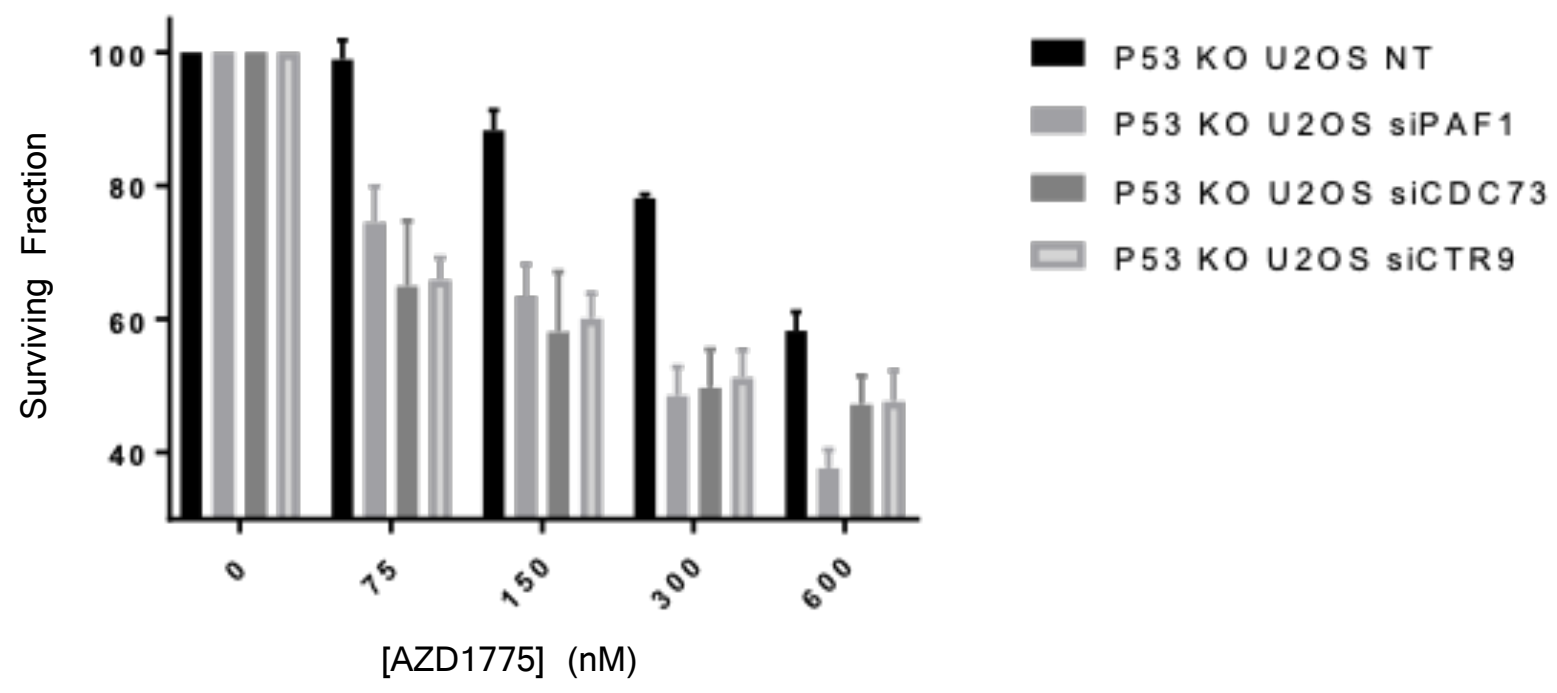

$\begin{array}{lll}\text { D) } & \text { SETD2 } & \text { SETD2 } \\ \text { WT U2OS } & \text { CRISPR } \\ & & \text { KO U2OS }\end{array}$

E)

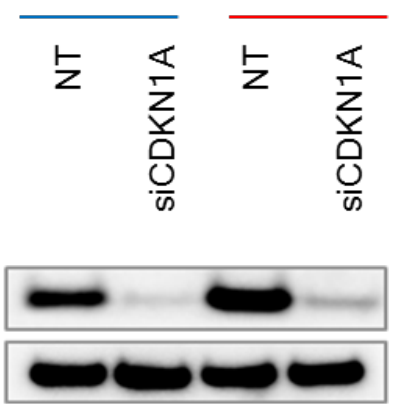

p21

Tubulin

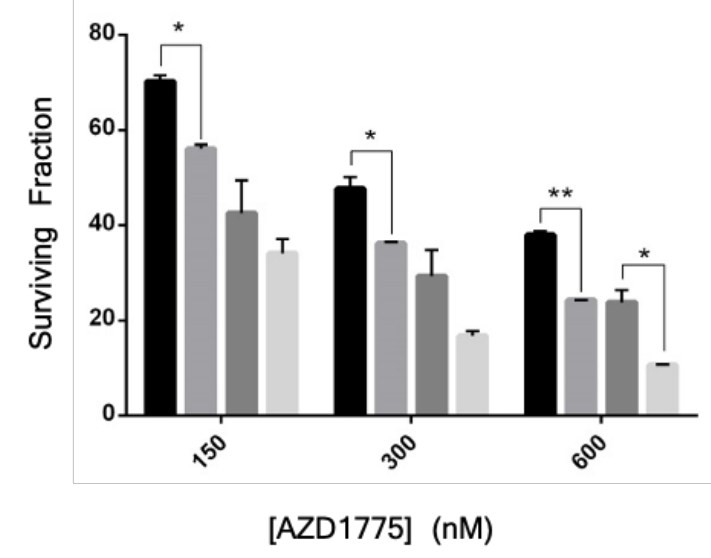

SETD2-WT NT

SETD2-WT SICDKN1A SETD2-MUT NT

SETD2-MUT SICDKN1A 


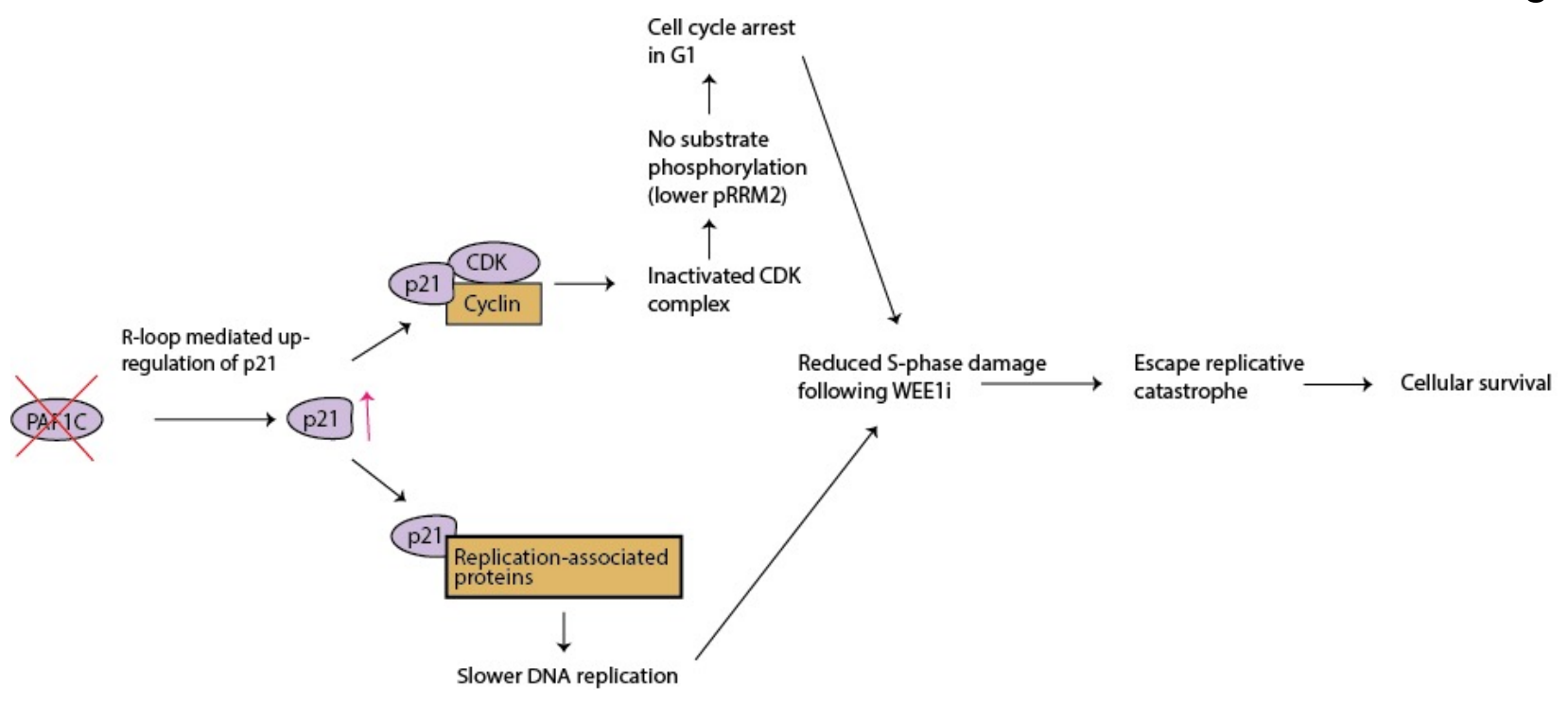


A)

$\begin{array}{ll}\text { SETD2 } & \text { SETD2 } \\ \text { WT U2OS } & \text { CRISPR } \\ & \text { KO U2OS }\end{array}$

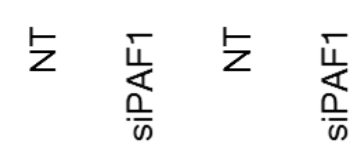

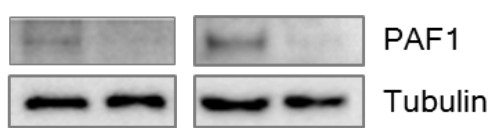

B)

Supplemental Figure 1

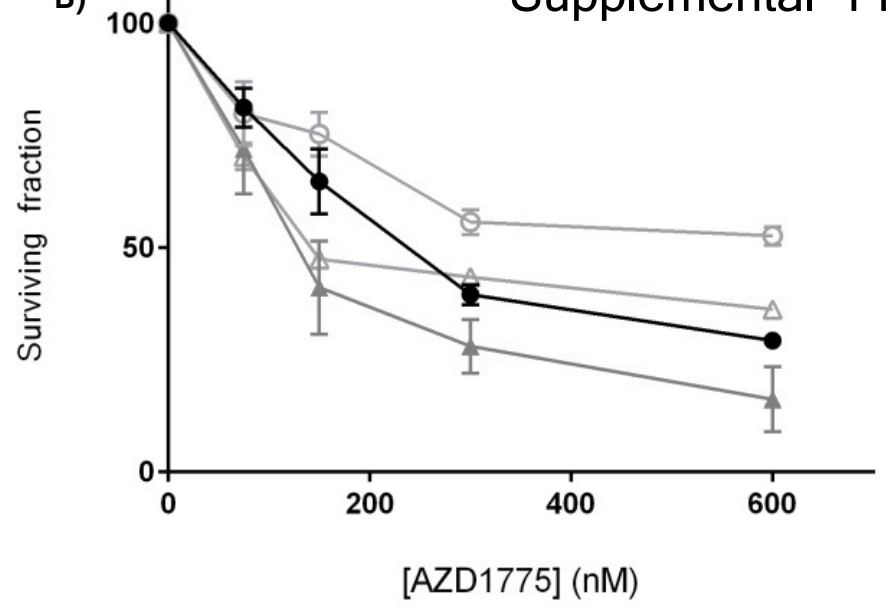

SETD2 WT

$\rightarrow$ NT

$\ominus \operatorname{siPAF} 1$

SETD2 MUT

$\pm \mathrm{NT}$

$\triangle \operatorname{siPAF} 1$

C)

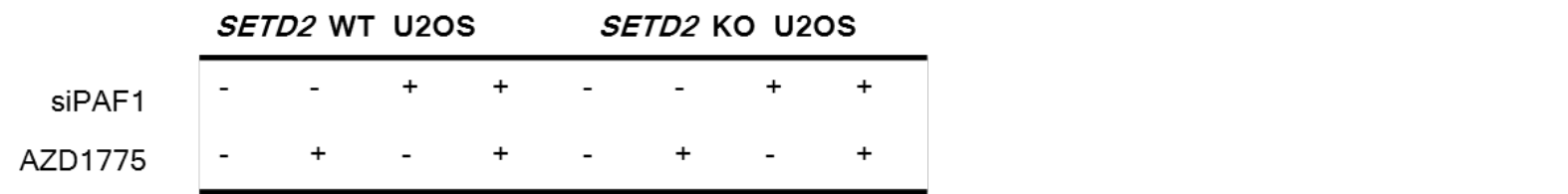

Cleaved PARP

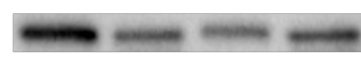

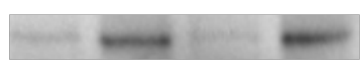

Procaspase 3

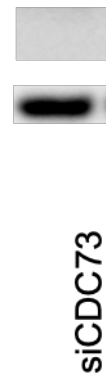

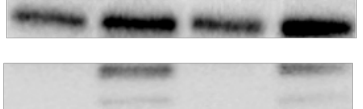

Cleaved caspase 3

Actin

D)

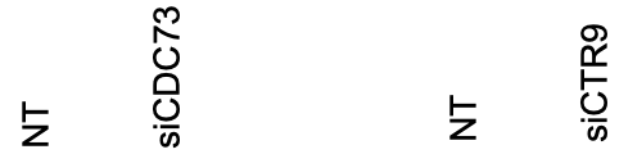
CDC73
CTR9
Tubulin
Tubulin 
A)

B)

AZD1775

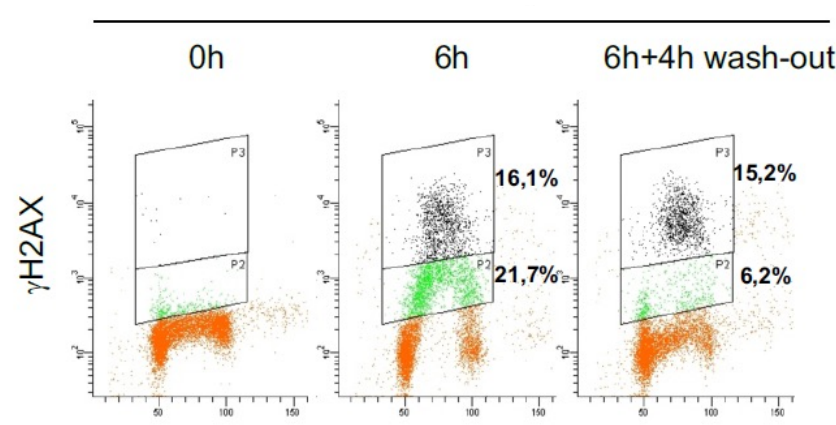

Supplemental Figure 2

\section{AZD1775}

$+2 \mathrm{~h}$ wash-out
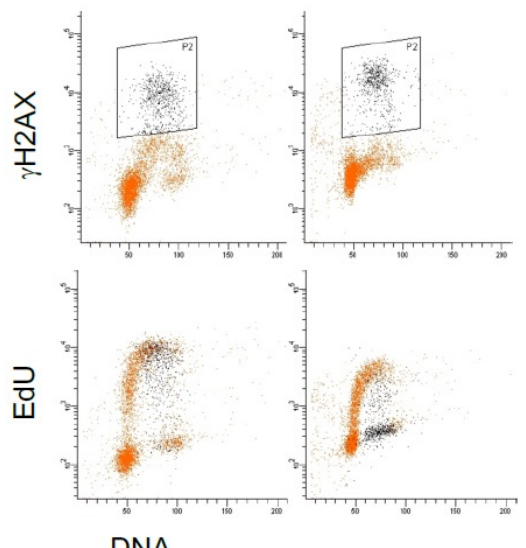

C)
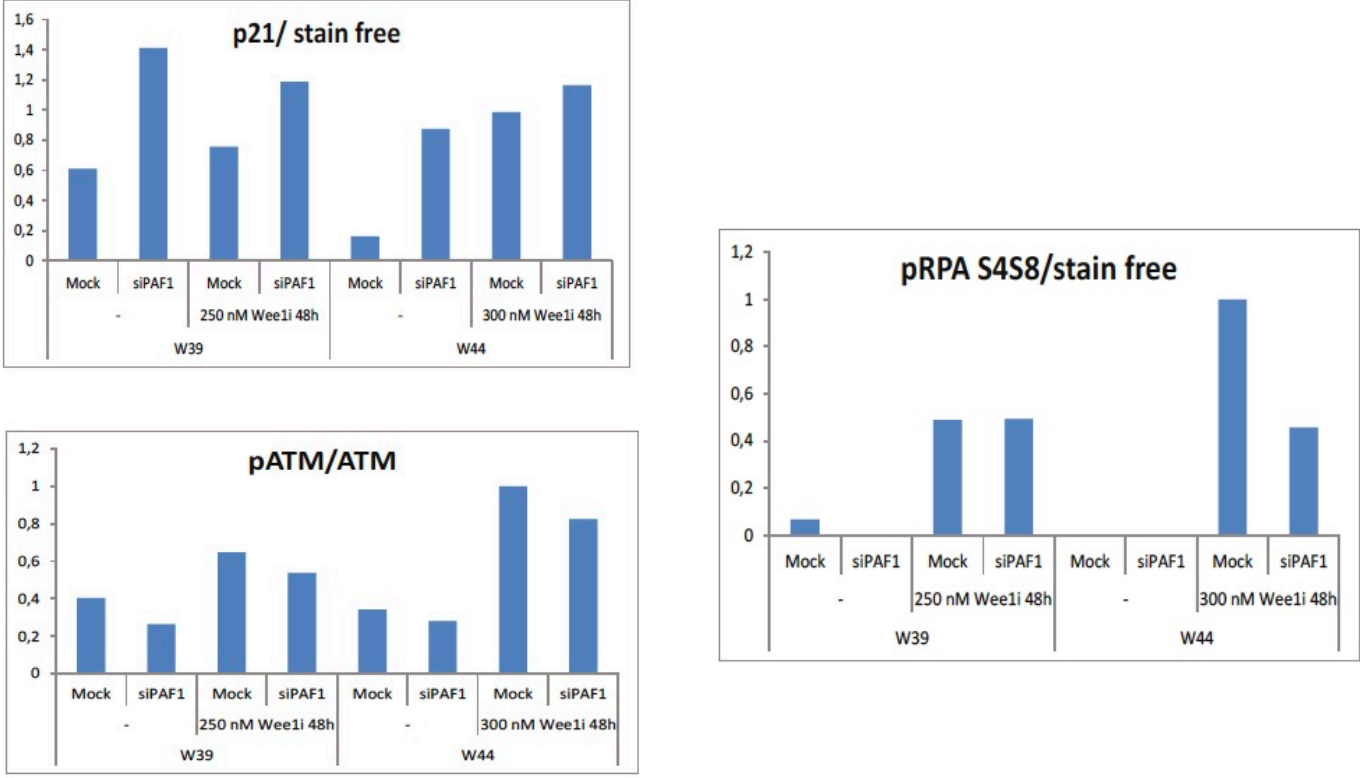
A) $\quad$ G1/S sync.

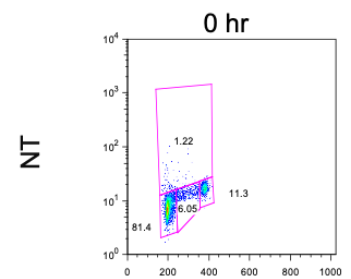

G1: $81.4 \%$

S: $1.22 \%$

G2/M: $11.3 \%$

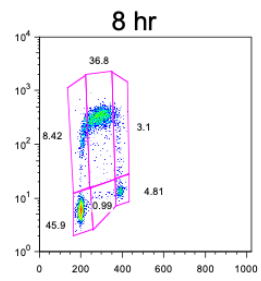

Supplementa

G1: $45.9 \%$

Mid-S: $36.8 \%$

Late-S: $3.1 \%$

G2/M: $11.3 \%$

G1: $75 \%$

S: $1.13 \%$

G2/M: $13 \%$

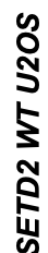

$\stackrel{N}{0}$
0
$\frac{0}{n}$
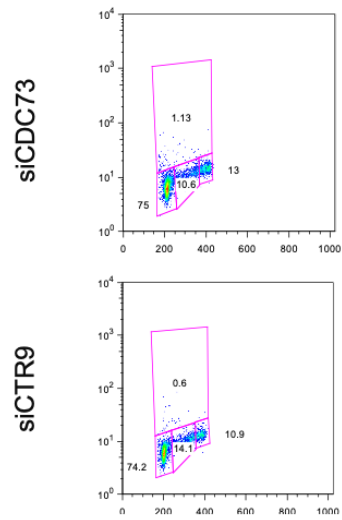

G1: $74.2 \%$

S: $0.6 \%$

G2/M: $10.9 \%$

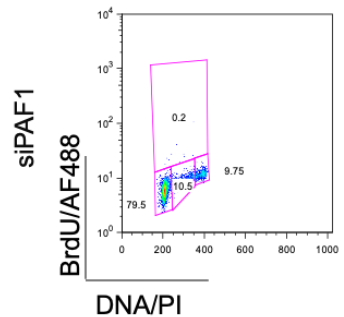

G1: $81.4 \%$

S: $1.22 \%$

G2/M: $11.3 \%$

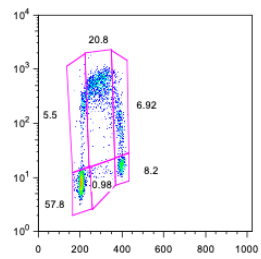

G1: $57.8 \%$

Mid-S: $20.8 \%$

Late-S: $6.92 \%$

G2/M: $8.2 \%$

G1: $50.9 \%$

Mid-S: $25.3 \%$

Late-S: $7.04 \%$

G2/M: $10.4 \%$

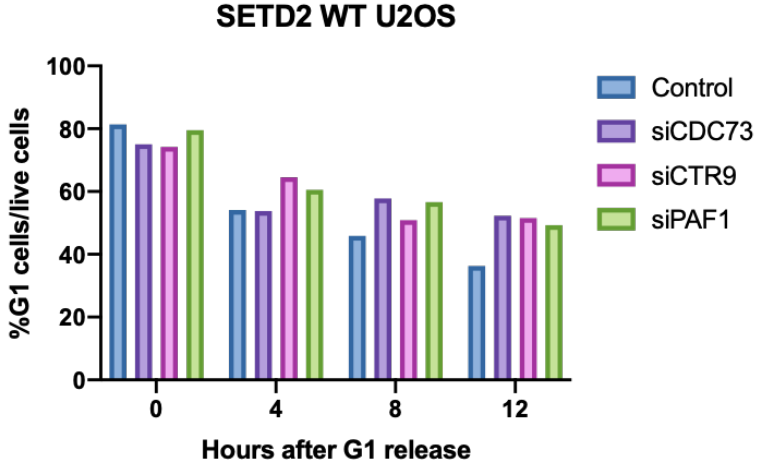

G1: $56.6 \%$

Mid-S: $25.2 \%$

Late-S: $4.29 \%$

G2/M: $6.3 \%$

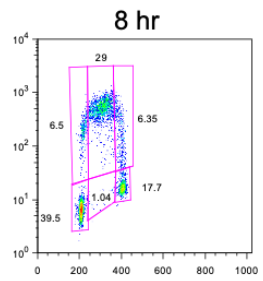

G1: $39.5 \%$

Mid-S: $29 \%$

Late-S: $6.35 \%$

G2/M: $17.7 \%$

S. $0.52 \%$

々
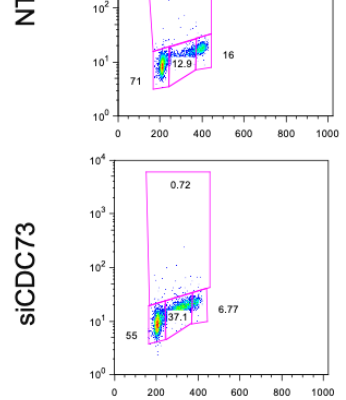

G1: $55 \%$

S: $0.72 \%$

G2/M: $6.77 \%$

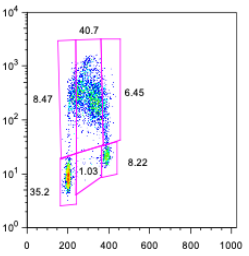

G1: $35.2 \%$

Mid-S: $40.7 \%$

Late-S: $6.45 \%$

G2/M: $8.22 \%$

G1: $60.2 \%$

S: $0.69 \%$

G2/M: $5.75 \%$

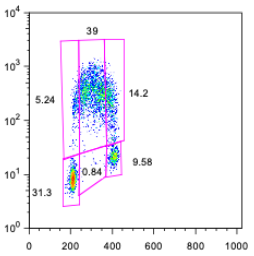

G1: $31.3 \%$

Mid-S: $39 \%$

Late-S: $14.2 \%$

G2/M: $9.58 \%$

G1: $51.9 \%$

S: $1.3 \%$

G2/M: $7.02 \%$

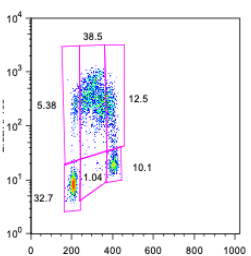


SETD2 CRISPR KO U2OS NT DMSO

SETD2 CRISPR KO U2OS SiCDKN1A

DMSO

SETD2 CRISPR KO U2OS SiPAF1

DMSO

SETD2 CRISPR KO U2OS siPAF1 + siCDKN1A DMSO
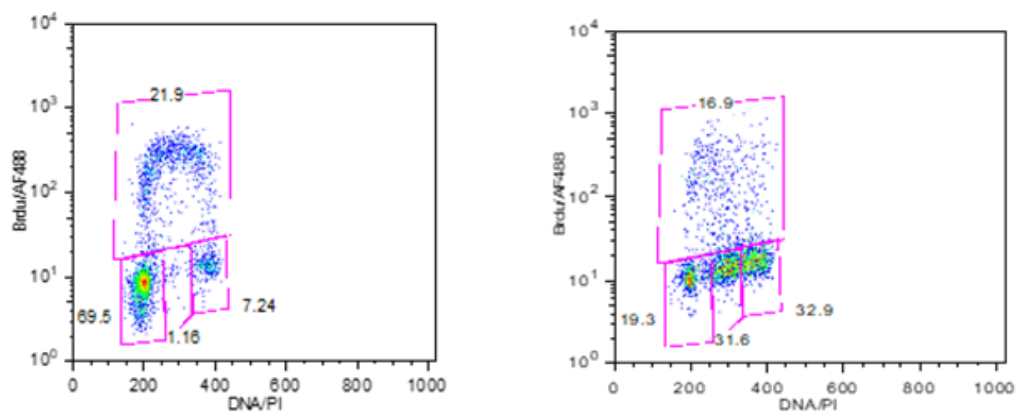

SETD2 CRISPR KO U2OS NT

AZD1775

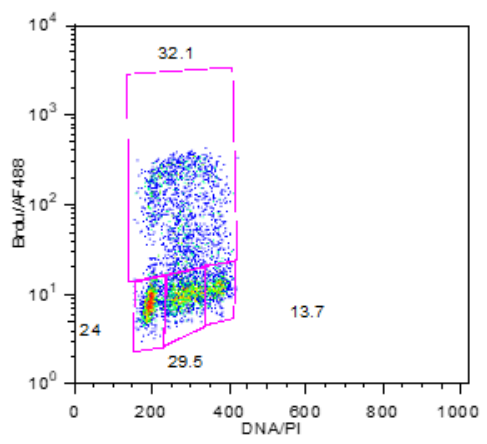

SETD2 CRISPR KO U2OS SiCDKN1A

AZD1775

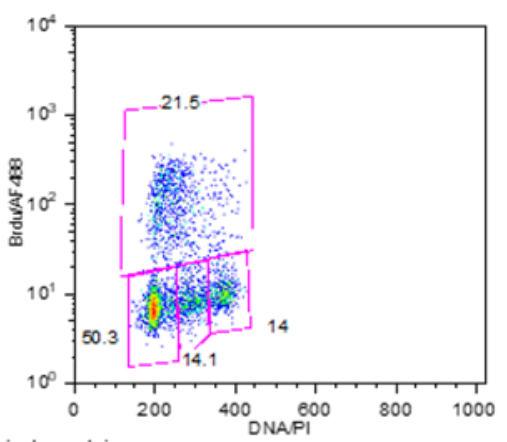

SETD2 CRISPR KO U2OS SIPAF 1

AZD1775

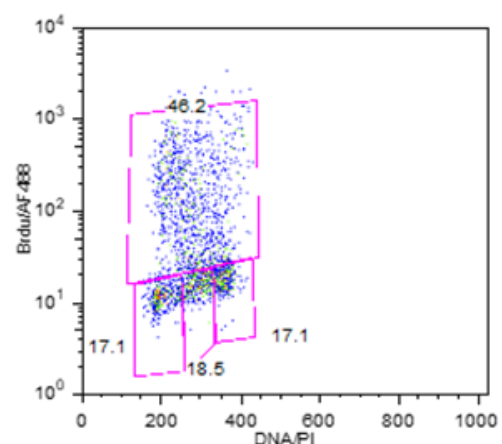

SETD2 CRISPR KO U2OS siPAF1 + siCDKN1A AZD1775 
DMSO
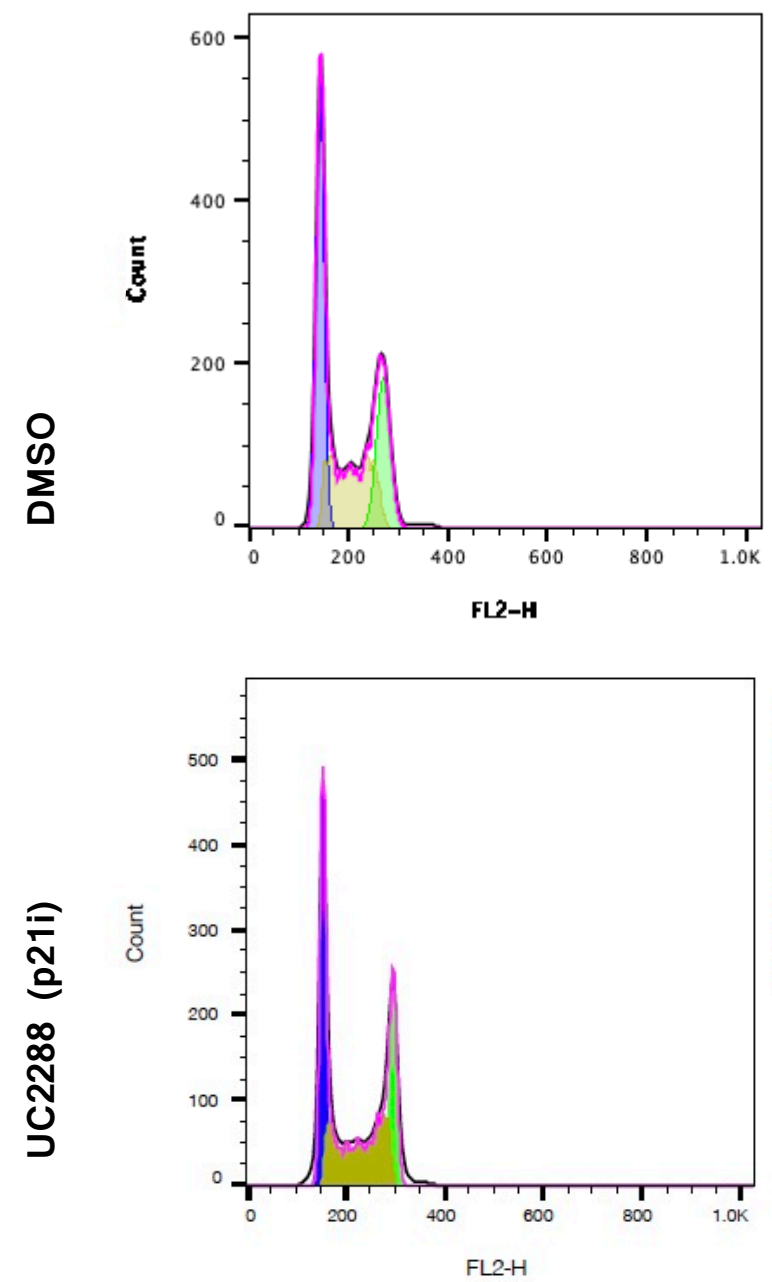

C)

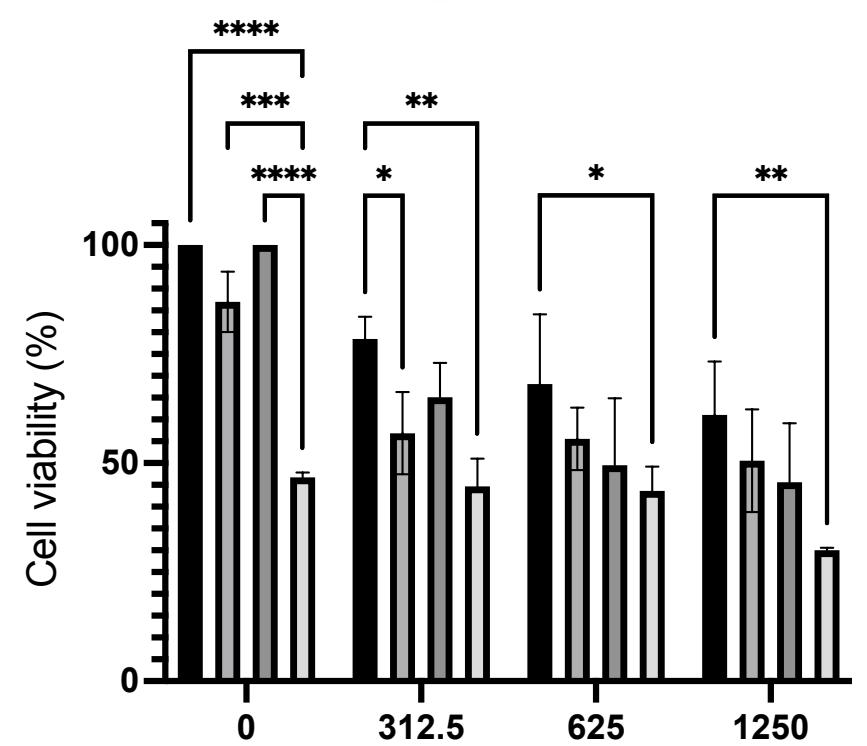

RMSD : 1.38

\%G1 : 41.5

$\% \mathrm{~S}: 33.7$

\%G2 : 23.3

G1 Mean : 142

G2 Mean : 268

G1 CV : 8.03

G2 CV : 7.28

\% less G1 : 0.79

\% greater G2 : 1.56

RMSD : 1.59

$\% \mathrm{Gl}: 33.6$

$\% \mathrm{~S}: 39.8$

$\%$ G $: 21.1$

Gl Mean : 155

G2 Mean : 298

$\mathrm{Gl} \mathrm{CV}: 5.28$

G2 CV : 3.49

\% less $\mathrm{Gl}: 2.30$

\% greater G2 : 3.16
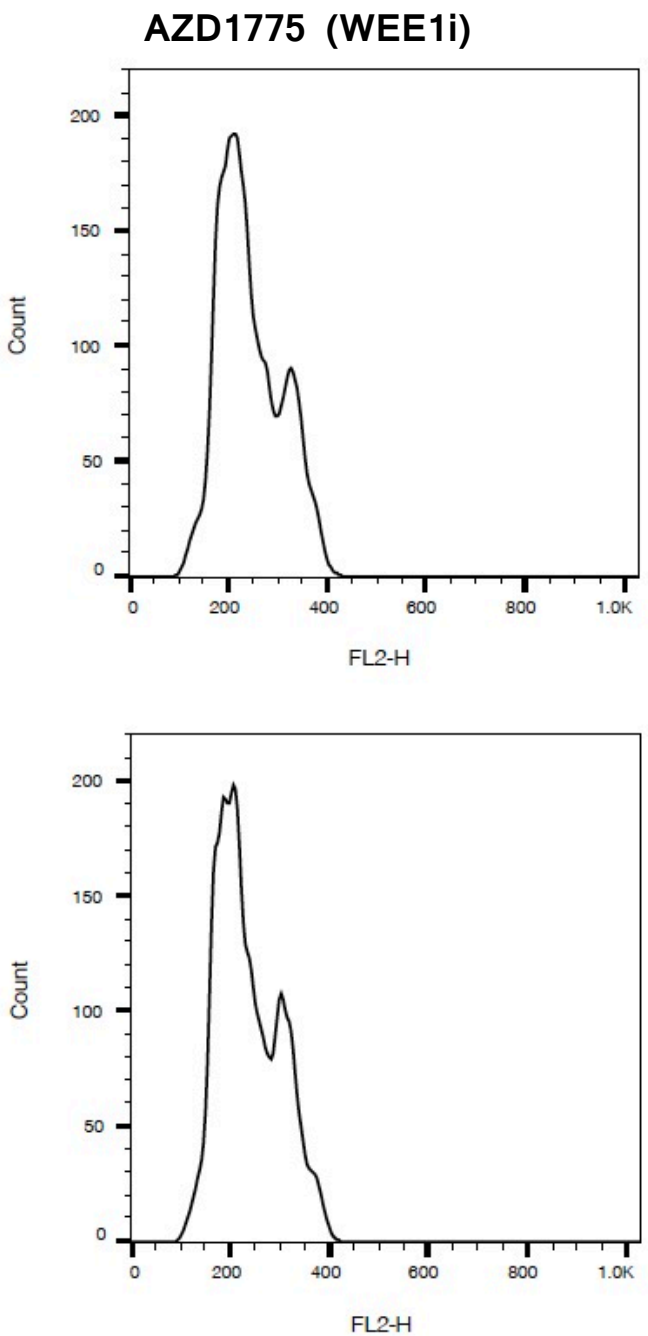

SETD2-WT AZD1775

口 SETD2-WT AZD1775 + UCN2288 (5 uM)

$\square$ SETD2-KO AZD1775

$\square$ SETD2-KO AZD1775 + UCN2288 (5 UM) 